\title{
Composition and evolution of the lithospheric mantle in central Spain: inferences from peridotite xenoliths from the Cenozoic Calatrava volcanic field
}

\author{
C. VILLASECA ${ }^{1 *}$, E. ANCOCHEA ${ }^{1}$, D. OREJANA ${ }^{1} \&$ T. E. JEFFRIES ${ }^{2}$ \\ ${ }^{1}$ Departamento de Petrología y Geoquímica, UCM-CSIC, Facultad de Geología, c/Jose \\ Antonio Novais, 2, 28040 Madrid, Spain \\ ${ }^{2}$ Department of Mineralogy, Natural History Museum, London SW7 5BD, UK \\ *Corresponding author (e-mail: granito@geo.ucm.es)
}

\begin{abstract}
Spinel lherzolite xenoliths from the Cenozoic Calatrava volcanic field provide a sampling of the lithospheric mantle of cen Spain. The xenoliths are estimate to originate from depths of $35-5 \mathrm{~km}$. Trace element content of clinopyroxene and Cr-mumber in spinel indicate low degrees of partial melting $(\leq 5 \%)$ of the xenoliths. Although a major element whole-rock model suggests wider degrees of meling, the Calawava perido chemistryindicates a moderately fervile mantle beneath central Spain. Calawava peridote xenoliths bear evidence for interaction with two different metasomatic agents. The enrichment in LREE (light rare earth element), Th, $\mathrm{U}$ and $\mathrm{Pb}$, and the negative anomalies in $\mathrm{Nb}-\mathrm{Ta}$ in clinopyroxene and amphibole from xenoliths of El Aprisco, indicate that the metasomatic agent was probably a subduction-related melt, whereas the enrichment in MREE in clinopyroxene from xenoliths of the Cerro Pelado centre suggests an alkaline melt similar to the host undersaturated magmas. These metasomatic agents are also consistent with the chemis of interstial glasses found in xenoliths of the two volcanic centres. Differences in metasomatism but also in mantle composition is supported by $\mathrm{Sr}-\mathrm{Nd}$ whole-rock data, which show a more radiogenic nature for $\mathrm{Sr}$ isotopes of samples from the El Aprisco centre

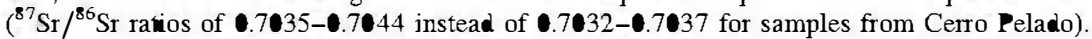
The riming of the subucton-relate metasomatic stage is unconstrained, although the Calava intraplate volcanism in udes an old Variscan lithospheric section reworked during the converging plate system affecting SE Iberia in the Teriary. The presence of wehrlite types within the Calarava peridote xenoliths is here interpreted as a reaction of host lherzolites with silica-undersaturated silicate melts that could be related to the Calatrava alkalinemagmatism. The $\mathrm{Sr}-\mathrm{Nd}$ isotopic composition of Calatrava peridotes plot within the European asthenospheric reservoir (EAR) mantle, these values represent more enriched signatures than those found in the other Spanish Cenozoic alkaline province of Olot.
\end{abstract}

Studies of ultramafic xenøliths exhumed by Cenzoic velcanic activity have previded substantial information regarding the nature of the subcontinental lithospheric mantle (e.g. Nix॰n 1987; Downes 2001). In the Iberian peninsula three main Cen z velcanic fields have provided significant mantlederived xenelith suites since studies from the last century: SE Spain (Ossan 1889), Olet (San Miguel de la Cámara 1936) and Calatrava (Ancechea \& Nix॰n 1987) (Fig. 1). Scarce ultramafic xeneliths have als been described in the Cofrentes velcanic area (Ancechea \& Nixøn 1987; Seghedi et al. 2002), and mantle-derived xeneliths have been found in Upper Permian subvelcanic dykes of the Spanish Central System, although they represent mafic-ultramafic cumulates instead of real mantle peridotitic fragments (Orejana et al. 2006; Villaseca et al. 2007; Orejana \& Villaseca 2008).
In this work we study the chemical compesition -f the Calatrava sample suite, including majer and trace elements for the constituent minerals, and majer, trace elements and $\mathrm{Nd}$ and $\mathrm{Sr}$ is topes for whøle rocks. As for many spinel lherz lite xenølith suites, our data indicate the decoupling of chemical features caused by melt extraction during partial melting and subsequent metasematism. This study, together with that of Bianchini et al. (2010), are the first attempts to characterize the subcentinental mantle beneath central Spain.

\section{Geological setting}

The Calatrava velcanic field comprises more than 200 velcanic centres in an area of around $5500 \mathrm{~km}^{2}$ (Ancechea 1982). The volcanic field is exclusively formed by monogenetic edifices, 
(a)

Neogene-Quaternary volcanics Post-Alpine sedimentary basins Alpine foldbelts \# Hercynian Massif

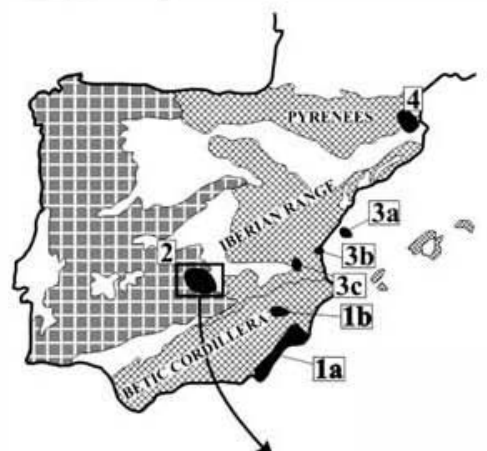

(b)

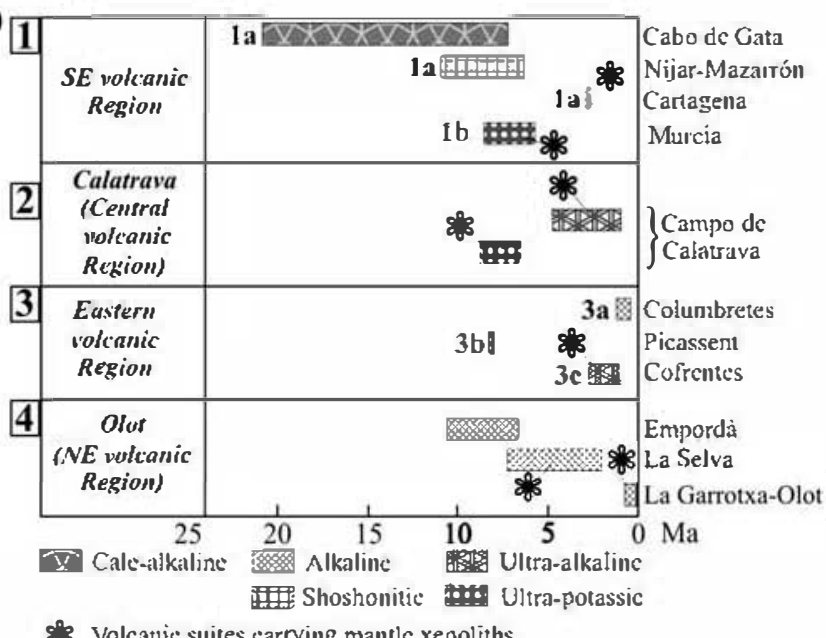

(c)

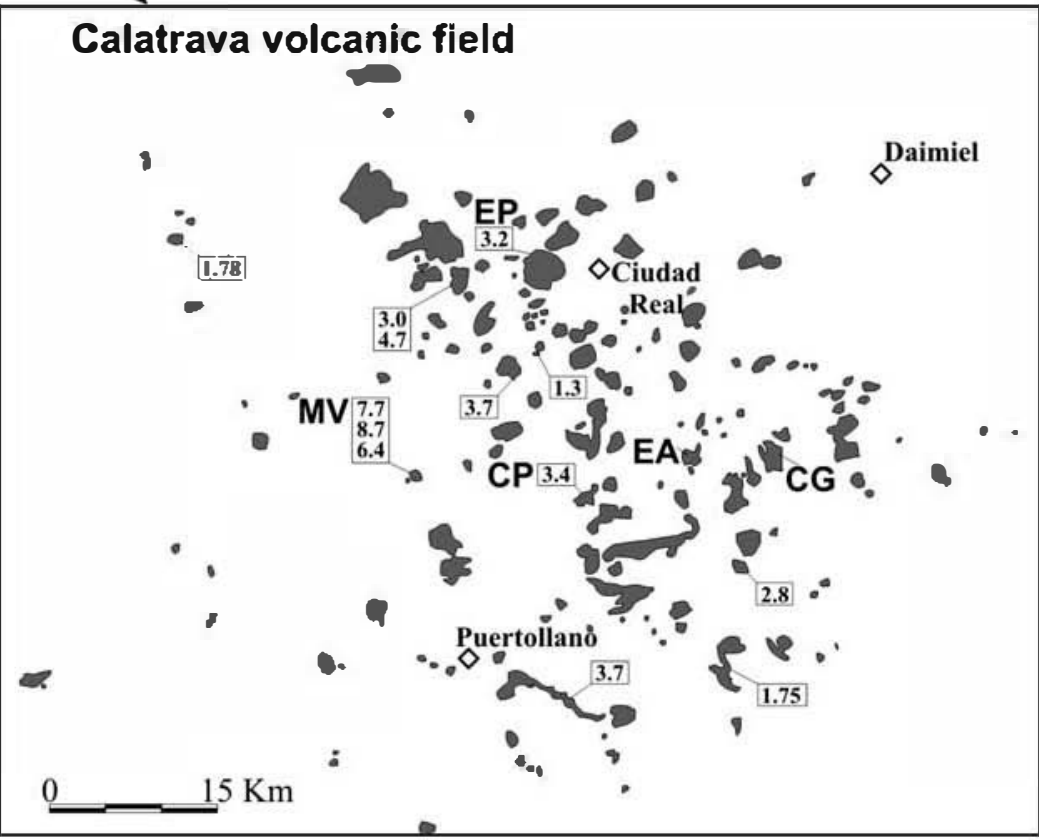

Fig. 1. (a) Sketch map $\bullet$ the Iberian Peninsula shøwing the løcation $\bullet$ the Cen॰zoic v•lcanic fields. (b)

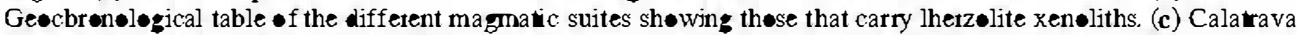

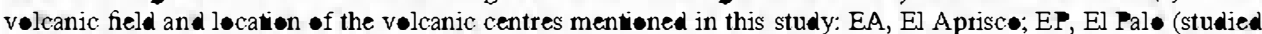

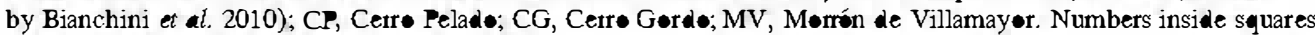
refer to geocbronølogical data (Ancøchea 204).

suggestive of small and shert-lived shallow magma chambers. Most edifices are strombelian cones but more than 50 hydromagmatic tuff rings or maars have been alse described. Both types of velcanic edifices yield periderite xenoliths. A first miner ulrapotassic event at around 8.7-6.4 Ma was followed by the eruption of alkaline basalts, basanites, and $\bullet$ livine nephelinites and melilitites from 3.7 t• arøund circa $0.7 \mathrm{Ma}$ (Ancechea 2004). Xenoliths studied in this work have been sampled from the pyroclastic depesits of two undersaturated alkaline basalic velcanoes: the Cerre Pelade centre $\left(38^{\circ} 48^{\prime} 32^{\prime \prime} \mathrm{N}, 3^{\circ} 54^{\prime} 56^{\prime \prime} \mathrm{W}\right)$, an $\bullet$ livine nephelinite scoria cone, and in El Aprisce $\left(38^{\circ} 50^{\prime} 05^{\prime \prime} \mathrm{N}\right.$; $3^{\circ} 5{ }^{\circ} \boldsymbol{0}^{\prime}(\mathrm{W})$, an $\bullet$ livine melilite maar. Smaller lherzelite xenøliths appear in other volcanic 
centres, including that of the early ultrapotassic event (El Mørrón de Villamayør, Cerrø Gørde, El Pale, etc.) (Fig. 1), but they have not been sampled for this study.

The similarity in normalized trace element patterns and the $\mathrm{Sr}-\mathrm{Nd}-\mathbf{P b}$ isøt॰pic hømøgeneity of the primary alkaline and ultralkaline basic magmas suggest that the Calatrava basaltic suites are derived from different degrees of partial melting of an enriched, but relatively hømøgeneøus mantle søurce (Ancœchea 1982; Cebriá \& LópezRuiz 1995). The isøt•pic composition suggests a HIMU-like reserv ir similar to that defined for the European asthenospheric mantle (Granet et al. 1995). An enriched asthenospheric reservoir (EAR) is in agreement with the suggested presence of garnet and phlogepite within mantle sources based on trace element geøchemistry of Calatrava vølcanics (e.g. Ancochea 1982; López-Ruiz et al. 2002).

The Calatrava velcanic field is a typical intracontinental zone within the Neogene-Present western and central European province. Similar to the NE

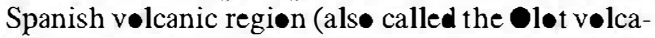
nic field), this area is located in the limit of small

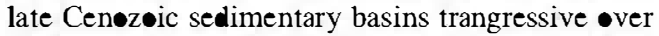
Variscan terranes. V•lcanic vent distribution does n॰t f॰llow a clear spatial pattern and their geodynamical setting is contrøversial, with theories inveking a gigantic megafault system affecting the western Mediterranean Eurøpean block (López-Ruiz et al. 2002) •r velcanic clustering related to asthen spheric mantle upwelling (høt-sp七 or diapir instabilities) in a pre-rifting stage (Ancechea 1982; Lépez-Ruiz et al. 1993).

\section{Analytical methods}

The majør element mineral composition has been analysed at the Centr• de Micrescøpía Electrónica 'Luis Bru' (Cømplutense University of Madrid) using a Jeøl JXA-8900 M electrøn micrøpr be with four wavelength-dispersive spectrøeters. Analytical conditions were an accelerating voltage -f $15 \mathrm{kV}$ and an electron beam current of $20 \mathrm{nA}$, with a beam diameter of $5 \mu \mathrm{m}$. Elements were counted for $10 \mathrm{~s}$ on the peak and $5 \mathrm{~s}$ on each background position. The measurement of $\mathrm{Ca}$ in -livine was repeated in some crystals with high content of this element (samples 65290 and 72674 ) using EMP conditions of $20 \mathrm{kV}, 50 \mathrm{nA}$ with counting times of $60 \mathrm{~s}$. T॰ reduce alkali loss during glass analysis, we lowered the beam current to $10 \mathrm{nA}$ and the beam was def $\bullet$ cused to $10 \mu \mathrm{m}$. Corrections were made using an on-line ZAF method. Detection limits are $\mathbf{0 . 0 2} \mathrm{wt} \%$ for $\mathrm{Al}, \mathrm{Na}, \mathrm{K}$ and $\mathrm{P}, 0.03$ wt\% for $\mathrm{Ti}, \mathrm{Fe}, \mathrm{Mn}, \mathrm{Mg}, \mathrm{Ni}$ and $\mathrm{Cr}$, and 0.04 wt\% for Si.
We have determined the in situ concentrations of 30 trace elements (REE, Ba, Rb, Sr, Th, U, Nb, Ta, $\mathbf{P b}, \mathrm{Zr}, \mathrm{Hf}, \mathrm{Y}, \mathrm{Sc}, \mathrm{V}, \mathrm{C} \bullet, \mathrm{Zn}$ and $\mathrm{Cr}$ ) in clinepyrex-

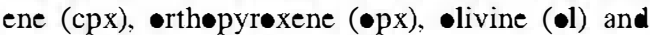

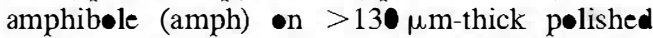
sections using laser ablation (LA-ICP-MS) at the Natural Histøry Museum of Løndon using an Agilent 7500CS ICP-MS coupled to a New Wave UP213 laser source (213 nm frequency-quadrupled Nd-YAG laser). The counting time f $\bullet$ r ne analysis was typically $90 \mathrm{~s}$ (40 $\mathrm{s}$ measuring gas blank to establish the background and $50 \mathrm{~s}$ for the remainder -f the analysis). The diameter of the laser beam was arøund $50 \mu \mathrm{m}$. The NIST 612 glass standard was used to calibrate relative element sensitivities for the analyses of the silicate minerals. Each analysis

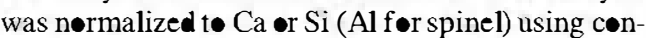
centrations determined by electron micrøprøbe Detection limits for each element were in the range of $0.01-0.06 \mathrm{ppm}$, except for $\mathrm{Sc}$ and $\mathrm{Cr}$ $(0.11$ and 0.73 ppm, respectively).

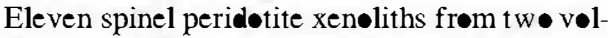
canic centres (El Aprisc $\bullet$ and Cerr $\bullet$ Peladø) were used in this investigation. The whole-røck majør and trace element composition was analysed at ACTLABS. The samples were melted using $\mathrm{LiBO}_{2}$ and disselved with $\mathrm{HNO}_{3}$. The selutions were analysed by inductively coupled plasma at mic emission spectrometry (ICP-AES) for major elements, whereas trace elements were determined by ICP mass spectrometry (ICP-MS). Uncertainties in major elements are bracketed between 1 and $3 \%$, except for $\mathrm{Mn}(5-10 \%)$ and $\mathbf{P}_{2} \mathbf{O}_{5}$ $(>10 \%)$. The precision of ICP-MS analyses at low concentration levels was evaluated from repeated analyses of the international standards BR, DR-N, UB-N, AN-G and GH. The precision for $\mathbf{R} b, \mathrm{Sr}$, $\mathrm{Zr}, \mathrm{Y}, \mathrm{V}, \mathrm{Hf}$ and most of the REE were in the range $1-5 \%$, whereas they range from 5 t॰ $10 \%$ for the rest of trace elements, including $\mathrm{Tm}$. S॰me samples had concentrations of certain elements below detection limits $\left(\mathrm{K}_{2} \bullet \mathbf{0 . 0 1 \%} ; \mathbf{R b} 1 ; \mathrm{Zr} 1\right.$;

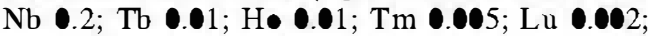

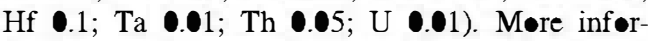
mation on the procedure, precision and accuracy -f ACTLABS ICP-MS analyses is available at www.actlabs.com.

$\mathrm{Sr}-\mathrm{Nd}$ isøtepic analyses were carried $\bullet$ ut at the

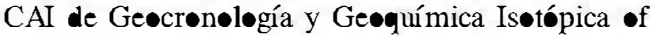
the Complutense University of Madrid, using an automated VG Sector 54 multicollector thermal ionization mass spectrometer with data acquired in multidynamic mode. Is topic ratios of $\mathrm{Sr}$ and Nd were measured on a subset of whole-rock powders. The analytical procedures used in this laboratory have been described elsewhere (Reyes et al. 1997). Repeated analysis of NBS 987 gave ${ }^{87} \mathrm{Sr} /{ }^{86} \mathrm{Sr}=\mathbf{0 . 7 1 0 2 4 9} \pm 30(2 \sigma, n=15)$ and 
for the JM Nd standard the ${ }^{143} \mathrm{Nd} /{ }^{144} \mathrm{Nd}=$ $0.511809 \pm 20(2 \sigma, n=13)$. The $2 \sigma$ error on the $\varepsilon(\mathbb{N d})$ calculation is \pm .4 .

\section{Petrography and mineral chemistry}

The studied Calatrava mantle xenoliths are rounded medium-size samples (from 5 to $45 \mathrm{~cm}$ in diameter) that show ne evidence of alteration or host basalt infil tation. Xenoliths equilibrated in the spinel peridotite stability field and display a wide mal vari-

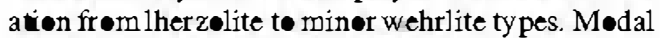
composition was determined by mass-balance calculations from the main minerals and the major element compositions of the whole rocks, using the least-squares inversion method of Albarède (1995). Within the 11 analysed rock samples, 10 are lherzolites and only one is a wehrlite (sample 72674) (Fi. 2). Mantle xenoliths from the E1 Aprisce centre tend to have orthopyroxene more abundant than clinopyroxene, whereas those from Cerr• Pelad• are more clinopyroxene-rich, even wehrlitic in composition (Fit. 2). The lher zoliticharzburgic mantle xeneliths from lot (Banchini et al. 2007; Galán et al. 2008) have been plotted for comparisen, and are similar in medal composition to those from SE Spain (Tallante: Beccaluva et al. 2004) (not shown). Calatrava therzelites are richer in clinopyroxene and perer in $\bullet$ livine than -ther Spanish mantle xenolith suites (Fit. 2). Scarce phlogepite-rich clinopyroxenites (Elimmerite varieties) have been found at Cerr Pelad• (Ancechea \& Nixon 1987) but they have not been sampled for this study.

Although in accessory amounts, the studied peridetite xenoliths usually have interstitial volatile-rich phases indicative of modal metas matism: amphibele in samples from the E1 Aprisce centre, and phlogepite from those of the Cerro Pelad - maar. Only one xenolith is an anhydrous lherzolite (sample 72690 from El Aprisco). The wehrlite 72674 shows race amounts of phlogepite included in clinepyroxene. Altheugh peridetite xen॰liths from Cerr- Pelad• with both hydrous minerals, amphibole and phlogepite, have been described previously (Ancechea \& Nixøn 1987) we did not find this type.

Most peridotite xenoliths have a coarse-grained texture of protegranular aspect, defined by a grain size greater than $2 \mathrm{~mm}$ and commonly equigranular (Fic. 3a). Some porphyroclastic textures or more

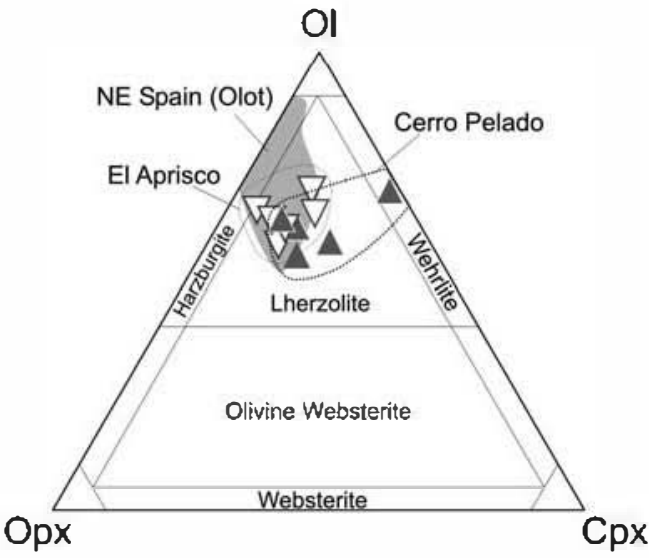

Fig. 2. Modal proportions of the studied Calatrava mantle xenoliths calculated using the mass-balance method of Albarèle (1995). The modal compositional field from mantle xenoliths from NE Spain (Olot) is taken from Bianchini et al. (2007) and Galán et al. (20)8)

inequigranular varieties als appear, in which -livine or orth $\bullet$ pyroxene are commonly the porphyroclasts. Grain boundaries are usually curvilinear defining mosaic or riple-junction textures. No phase layering, foliation or lamination have been found.

- livine crystals may have different sizes even in a single sample. Some fine-grained interstitial crystals, spatially related to spinel microagzegates or spinel coronas as described below, have been considered to be of second zeneration. Both orthopyroxene and clinopyroxene show mutual lamellae, and commenly a second superimposed spinellamel1ae. Spinel $\bullet$ ccurs as discrete, dispersed interstial grains that usually show some fine-grained polycrystalline corenas of amphibele (only in the $\mathrm{El}$

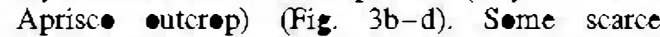
spinel-pyroxene-amphibele symplecte has been als observed, but the clearly hydrous character of the corona rejects the possibility of a reaction between pre-existing garnet and matrix livine, as sugzested in other spinel symplectitic lherzelite xeneliths (Ackerman et al. 2007) (Fic. 3c). Some spinel grains have sieve textures defined by a partial corona of a new fine-grained spinel- 2 associated to vesicular glass and second-zeneration -livine (Fi 3f). These textural features have been interpreted as re-equilibrations or reactions with a

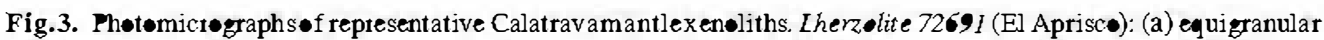

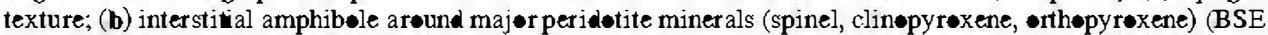

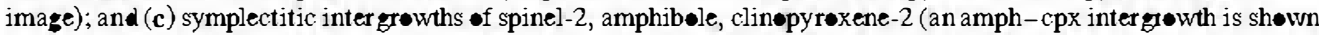

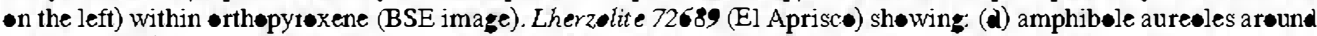
spinel; and (e) a complex reaction zone showing the breakdown of spinel to clinopytoxene-2, livine-2 and vesicular 

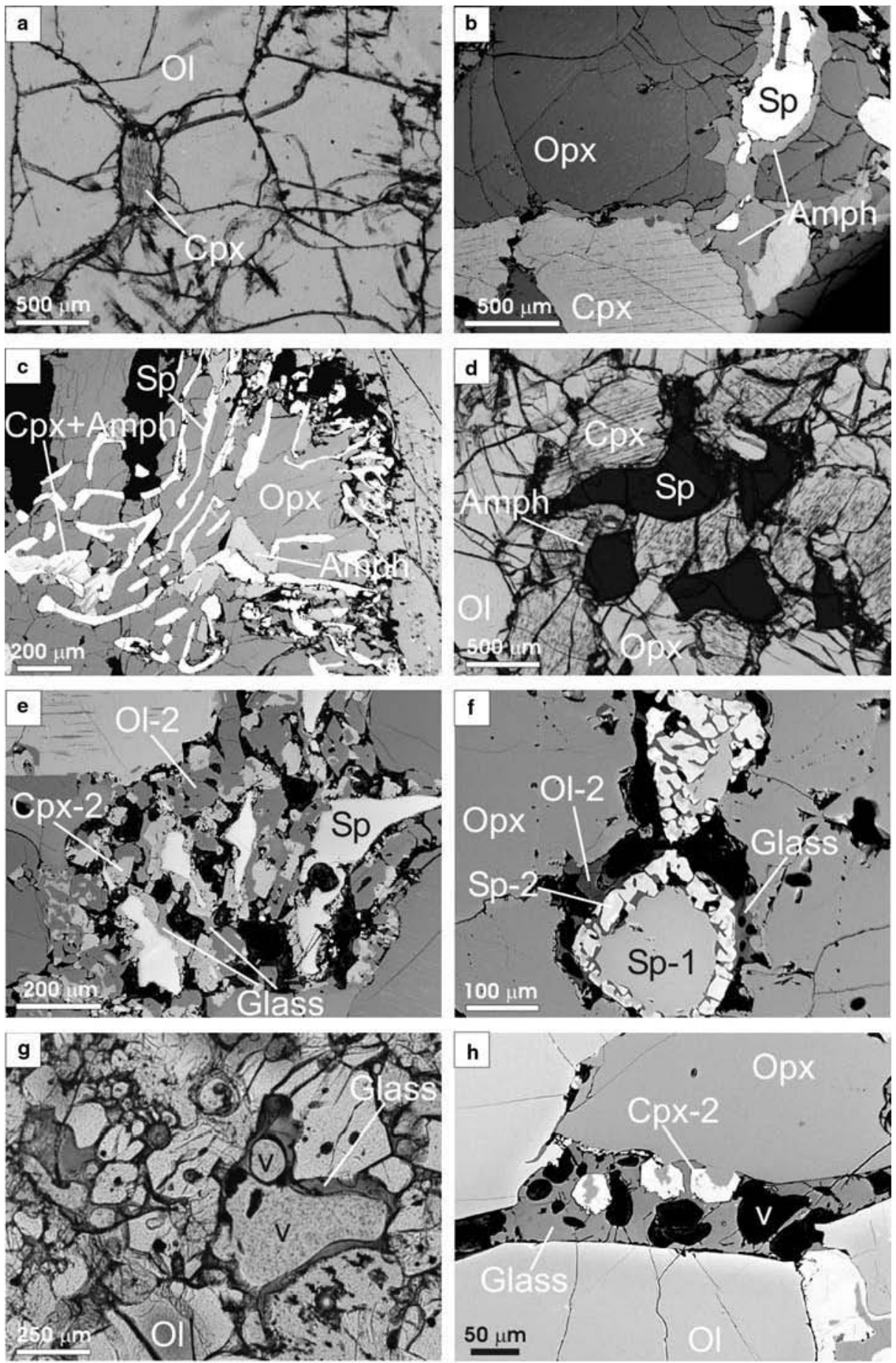

Fig.3. (Continued) glass (BSE image). Lherzolite 65290 (Certo Pelado): (f) reaction zones around primary spinel (Sp-1) compesed of cellular spinel-2, mictogranula relivine-2 and vesicular lass (BSE image). Wehrlite 72674 (Cerr• Pelado): (g) highly vesicular interstitial lass (BSE image); and (h) vesicular lass vein with asseciated cpx-2 crystals

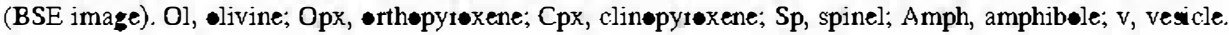


percelating intergranular melt (Shaw \& Dingwell 2008). In sample 65290 it is possible to see a complex corona of symplectitic spinel-2 and interstitial glass rimming primary spinel (Fig. 3f). Acicular pentlandite, interstitial to •livine, is very scarce in søme lherzolites.

Accessøry amøunts of hydrøus minerals (amphi-

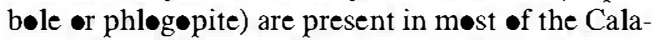
trava lherzolite xenoliths (except in anhydrous lherzelite 72690). They are mostly intergranular phases forming small anhedral crystals. Amphibøle in most lherzolites forms corønas arøund spinel grains or intergrøwths with clinøpyrøxene arøund spinel symplectites (Fig. $3 b-d$ ), in textures similar to those described in other peridotite xenolith suites (e.g. Coltorti et al. 2004, 2007b).

S॰me xen॰liths als• sh॰w interstitial brøwn glass with small vesicle or bubble-like structures (Fig. $3 e-h)$. Moreøver, some interstitial glasses appear as part of a complex reaction zøne that invelves many of the primary lherzelite minerals, but especially spinel, which is surrounded either by symplectitic intergrowths of new spinel- 2 and - livine-2 with interstitial glass (Fig. 3f) or by a micreaggregate of newly formed $\mathrm{cpx}-\bullet 1$-glass (Fig. 3e).

The wehrlite 72674 is porphyreclastic in texture

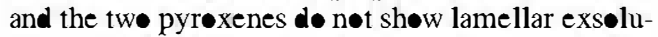
tion, as is typical in the lherzelite types. Wehrlite clinopyroxene shows a marked poikilitic texture with multiple glass, apatite, phlogopite and fluidrich inclusions. Olivine grains show lecally def ormation twins and most crystals have smoeth curvilinear boundaries. Only trace amounts of spinel (as micreinclusions in elivine and clinepyroxene) appear in this sample. The wehrlite als shows interstitial vesicular glass, which is mainly concentrated in the fine-grained section of the inequigranular texture, defining søme interconnected veining (Fig. 3g, h).

\section{Major element mineral composition}

The Calatrava hydrøus mantle xenøliths cønsist $\bullet$

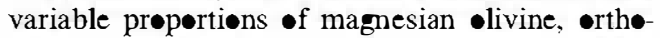
pyrøxene and clinøpyroxene, aluminøus spinel, and accessery amounts of calcic amphibøle or phlogøpite, the compositions of which are summarized in Table 1. All minerals are unzoned and homegeneous within a single crystal.

The Mg-number f $\bullet$ livine mostly ranges frøm 89.2 t• 91.5, althøugh neof $\bullet$ rmed varieties (•liv-2) and elivine from wehrlite 72674 show lower Mg-numbers (88.4 and 84.5-86.0, respectively). olivine-2 in lherzelites als• shøw slightly higher $\mathrm{Ca}$ and lower $\mathrm{Ni}$ content than $\mathrm{Mg}$-rich $\bullet$ livine (Table 1), features typical of metasomatism (e.g. Coltorti et al. 1999; Ionov et al. 2005). Olivine in the wehrlite shøws the highest $\mathrm{Ti}_{2}$ cøntent (up t• $0.09 \mathrm{wt} \%$ ), and high $\mathrm{Ca}$ and low $\mathrm{Ni}$ content (Table 1).

Orthøpyroxene has a similar range of $\mathrm{Mg}$ -

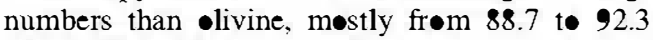
(Table 1), but it shøws a wider range in content of $\mathrm{Cr}_{2} \bullet_{3}(.13-0.52), \mathrm{Al}_{2} \bullet_{3}(3.00-5.74)$ and $\mathrm{Ca}$ (0.12-1.09), always in a commen range for abyssal peridetites (Bonatti \& Michael 1989). Cør-

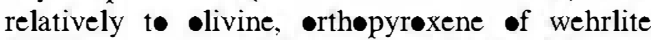
72674 shows lower Mg-numbers (85.0-86.6), and higher content of $\mathrm{TiO}_{2}(\mathbf{0 2 2 -} \mathbf{0 1}), \mathrm{Ca}(\mathbf{0 4}-$ $3.86)$ and $\mathrm{Na}_{2} \bullet(12-\mathbf{0}-25)$, than $\bullet$ rthøpyrøene frøm assøciated lherzolites (Fig. 4a). Lherzølite 72691 with the lowest averaged Al content of -rthopyroxene (and the highest $\mathrm{Mg}-\mathrm{Cr}$ values) could be the most depleted peridotite of the xenolith suite (Fig. 4a).

Clinøpyroxene alse shøws a wide range of Mg-numbers (87.6-92.7) with cpx-2 (neocrystals related t• intergranular reaction zønes) having low values (88.2-90.0) but variable $\mathrm{Al}-\mathrm{Ti}-\mathrm{Cr}$ content. For example, the cpx-2 analysis 71 in lher-

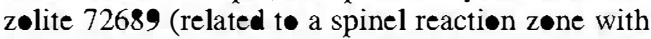
glass) shows the highest $\mathrm{Al}-\mathrm{Cr}-\mathrm{Ti}$ content of the whole analysed clinøpyroxene population (Fig. 4b).

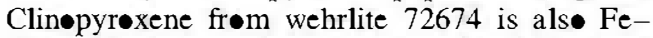
Ti-enriched, as are the $\bullet$ ther minerals of this xen lith (Fig. 4b, c) (Table 1). Lherzolite 72691 shows the lowest Al clinopyroxene, reinforcing the idea -f being the most depleted peridotite xen $\bullet$ lith.

Primary spinel has low Cr-numbers (frøm 8.3 to 10.8 ) and a narrow $\mathrm{Mg}$-number range $(\mathbf{0 . 7 5}-\mathbf{0 . 7 8})$ (Table 1). $\mathrm{Ti}_{2}$ content is generally løw, ranging from 0.01 to $0.16 \mathrm{wt} \%$. Spinel from the most depleted lherzelites (samples 72691 and 55570) shøws a wider range of cømp $\bullet$ ition, with relatively higher $\mathrm{Cr}$ values and lower $\mathrm{Al}$ content than spinel frøm associated lherzølites (Fig. 5). In lherzølite 55570 cores of large spinel crystals show the highest $\mathrm{Al}$ and $\mathrm{Mg}$ content (and the lowest $\mathrm{Cr}$ values) compared with rims or interstitial rods, which are asseciated with intergranular amphibøle. In fact, the smallest interstitial spinel crystals in lherzolite 55570 and the symplectitic spinel from lherzolite 72691 show the highest Cr-numbers (13.0-17.7) and the lowest $\mathrm{Al}_{2} \mathrm{O}_{3}$ contents (51$55 \mathrm{wt} \%) \bullet f$ the Calatrava lherzølites. These contents are similar to those of the sieved sp- 2 of lherz lites 65290 and 65298, which als• shøw high $\mathrm{TiO}_{2}$ cøntent (up t $0.44 \mathrm{wt} \%$ ), suggestive of a reaction with a Ti-rich metas-matic agent (Perinelli et al. $2008 b$ ) (Fig. 5). Residual spinel micr grains preserved as inclusions in majør minerals of wehrlite 72674 sh॰w the highest $\mathrm{Ti}$ and $\mathrm{Cr}\left(\mathrm{Ti}_{2}\right.$ up t॰ $3.0 \mathrm{wt} \%$ and Cr-numbers in the range of 52.454.1), and the lowest $\mathrm{Al}-\mathrm{Mg}$ content, of the studied peridetite xenøliths (Table 1). Owing t• 
Table 1. Major element composition of representative minerals from the Calatrava mantle xenoliths

\begin{tabular}{|c|c|c|c|c|c|c|c|c|c|c|}
\hline \multicolumn{11}{|c|}{ Representative olivines } \\
\hline \multirow[b]{2}{*}{ Sample } & \multicolumn{6}{|c|}{ El Aprisco } & \multicolumn{4}{|c|}{ Cerro Pelado } \\
\hline & $\begin{array}{c}55569 \\
\text { A39 }\end{array}$ & $\begin{array}{c}55570 \\
\text { A18 }\end{array}$ & $\begin{array}{l}72688 \\
\text { A142 }\end{array}$ & $\begin{array}{c}72689 \\
\text { A82 }\end{array}$ & $\begin{array}{c}72690 \\
\text { A38 }\end{array}$ & $\begin{array}{l}72691 \\
\text { A120 }\end{array}$ & $\begin{array}{c}65290 \\
\text { A23 }\end{array}$ & $\begin{array}{c}65298 \\
\text { A2 }\end{array}$ & $\begin{array}{c}65298 \\
21 \text { (oliv-2) }\end{array}$ & $\begin{array}{c}72674 \\
\text { A95 }\end{array}$ \\
\hline $\begin{array}{l}\mathrm{SiO}_{2} \\
\mathrm{TiO}_{2} \\
\mathrm{Al}_{2}{ }_{3} \\
\mathrm{Fe} \\
\mathrm{Mn} \\
\mathrm{Mg} \\
\mathrm{CaO} \\
\mathrm{Na}_{2} \\
\mathrm{~K}_{2} \\
\mathrm{Ni}^{-} \\
\mathrm{Cr}_{2}\end{array}$ & $\begin{array}{c}41.33 \\
\text { bdl } \\
\text { bdl } \\
9.63 \\
0.05 \\
49.60 \\
0.03 \\
\text { bdl } \\
0.01 \\
0.41 \\
\text { bdl }\end{array}$ & $\begin{array}{c}40.56 \\
\text { bdl } \\
\text { bdl } \\
9.18 \\
0.18 \\
49.54 \\
0.04 \\
0.02 \\
\text { bdl } \\
0.47 \\
0.08\end{array}$ & $\begin{array}{c}41.05 \\
\text { bdl } \\
\mathbf{0 . 0 2} \\
9.88 \\
.20 \\
49.61 \\
.09 \\
\mathbf{0 . 0 1} \\
\text { bdl } \\
0.22 \\
0.01\end{array}$ & $\begin{array}{c}39.97 \\
\text { bdl } \\
\text { bdl } \\
9.53 \\
0.18 \\
49.85 \\
0.04 \\
\text { bdl } \\
\text { bdl } \\
0.42 \\
0.07\end{array}$ & $\begin{array}{c}41.20 \\
0.01 \\
\text { bdl } \\
9.31 \\
\mathbf{0 . 0 6} \\
49.74 \\
\mathbf{0 . 0 2} \\
\mathbf{0 . 0 2} \\
\text { bdl } \\
\mathbf{0 . 3 3} \\
\mathbf{0 . 0 2}\end{array}$ & $\begin{array}{c}40.63 \\
\text { bdl } \\
\text { bdl } \\
8.66 \\
0.13 \\
50.68 \\
0.05 \\
0.01 \\
\text { bdl } \\
0.42 \\
0.03\end{array}$ & $\begin{array}{r}40.58 \\
0.02 \\
0.03 \\
10.40 \\
0.16 \\
49.01 \\
0.06 \\
\text { bdl } \\
\text { bdl } \\
0.21 \\
0.07\end{array}$ & $\begin{array}{c}40.67 \\
0.01 \\
\text { bdl } \\
9.29 \\
0.07 \\
50.43 \\
0.09 \\
0.01 \\
\text { bdl } \\
0.36 \\
0.03\end{array}$ & $\begin{array}{c}40.69 \\
0.01 \\
\text { bdl } \\
11.36 \\
0.13 \\
48.71 \\
0.13 \\
\text { bdl } \\
\text { bdl } \\
0.12 \\
0.04\end{array}$ & $\begin{array}{c}40.03 \\
0.03 \\
\text { bdl } \\
14.85 \\
0.12 \\
45.53 \\
0.09 \\
\text { bdl } \\
\text { bdl } \\
0.20 \\
\text { bdl }\end{array}$ \\
\hline $\begin{array}{l}\text { Total } \\
\text { XMg }\end{array}$ & $\begin{array}{r}101.06 \\
90.19\end{array}$ & $\begin{array}{l}99.98 \\
90.59\end{array}$ & $\begin{array}{r}101.09 \\
89.97\end{array}$ & $\begin{array}{l}99.99 \\
90.31\end{array}$ & $\begin{array}{r}100.71 \\
90.50\end{array}$ & $\begin{array}{r}100.58 \\
91.25\end{array}$ & $\begin{array}{r}100.49 \\
89.36\end{array}$ & $\begin{array}{r}100.92 \\
90.62\end{array}$ & $\begin{array}{r}101.15 \\
88.44\end{array}$ & $\begin{array}{r}100.91 \\
84.47\end{array}$ \\
\hline \multicolumn{11}{|c|}{ Representative orthopyroxenes } \\
\hline & \multicolumn{6}{|c|}{ El Aprisco } & \multicolumn{4}{|c|}{ Cerro Pelado } \\
\hline Sample & $\begin{array}{c}55569 \\
\text { A36 }\end{array}$ & $\begin{array}{c}55570 \\
\text { A32 }\end{array}$ & $\begin{array}{c}72688 \\
\text { A59 }\end{array}$ & $\begin{array}{c}72689 \\
\text { A76 }\end{array}$ & $\begin{array}{c}72690 \\
\text { A41 }\end{array}$ & $\begin{array}{c}72691 \\
\text { A65 }\end{array}$ & $\begin{array}{c}65290 \\
\text { A27 }\end{array}$ & $\begin{array}{c}65298 \\
\text { A23 }\end{array}$ & $\begin{array}{c}65298 \\
\text { A3 }\end{array}$ & $\begin{array}{c}72674 \\
\text { Al10 }\end{array}$ \\
\hline $\begin{array}{l}\mathrm{SiO}_{2} \\
\mathrm{TiO}_{2} \\
\mathrm{Al}_{2}{ }_{3} \\
\mathrm{Fe} \\
\mathrm{MnO} \\
\mathrm{Mg} \\
\mathrm{Ca} \\
\mathrm{Na}_{2} \\
\mathrm{~K}_{2} \\
\mathrm{Ni} \\
\mathrm{Cr}_{2}\end{array}$ & $\begin{array}{r}55.08 \\
0.15 \\
4.72 \\
6.26 \\
\mathbf{0 . 1 4} \\
32.80 \\
0.99 \\
\mathbf{0 . 1 1} \\
\text { bdl } \\
\mathbf{0 . 0 8} \\
\mathbf{0 . 3 3}\end{array}$ & $\begin{array}{r}54.47 \\
0.12 \\
5.00 \\
5.77 \\
0.14 \\
32.56 \\
1.10 \\
0.11 \\
\text { bdl } \\
\mathbf{0 . 1 4} \\
\mathbf{0 . 5 3}\end{array}$ & $\begin{array}{r}55.38 \\
0.03 \\
3.49 \\
6.54 \\
0.15 \\
33.39 \\
0.54 \\
0.02 \\
0.02 \\
\text { bdl } \\
0.18\end{array}$ & $\begin{array}{r}54.97 \\
0.06 \\
3.35 \\
6.41 \\
0.15 \\
33.94 \\
0.43 \\
\mathbf{0 . 0 3} \\
\text { bdl } \\
\mathbf{0 . 0 8} \\
\mathbf{0 . 2 3}\end{array}$ & $\begin{array}{r}54.73 \\
0.04 \\
5.42 \\
5.80 \\
0.12 \\
32.34 \\
1.37 \\
0.13 \\
\text { bdl } \\
0.08 \\
0.34\end{array}$ & $\begin{array}{r}54.71 \\
0.04 \\
4.08 \\
5.45 \\
0.13 \\
33.66 \\
0.62 \\
0.06 \\
\text { bdl } \\
0.15 \\
0.38\end{array}$ & $\begin{array}{r}54.19 \\
0.18 \\
5.74 \\
6.30 \\
\mathbf{0 . 1 4} \\
31.53 \\
1.04 \\
\mathbf{0 . 1 5} \\
\text { bdl } \\
\mathbf{0 . 0 4} \\
\mathbf{0 . 4 0}\end{array}$ & $\begin{array}{r}54.24 \\
0.07 \\
5.49 \\
6.11 \\
0.15 \\
32.96 \\
0.88 \\
0.12 \\
\text { bdl } \\
0.18 \\
0.35\end{array}$ & $\begin{array}{r}53.88 \\
\mathbf{0 . 0 8} \\
5.39 \\
6.24 \\
\mathbf{0 . 1 3} \\
32.81 \\
\mathbf{0 . 8 0} \\
\mathbf{0 . 1 5} \\
\text { bdl } \\
\mathbf{0 . 1 4} \\
\mathbf{0 . 3 3}\end{array}$ & $\begin{array}{r}53.64 \\
0.37 \\
3.94 \\
8.46 \\
0.15 \\
28.54 \\
3.86 \\
0.25 \\
\text { bdl } \\
\text { bdl } \\
\mathbf{0 . 4 4}\end{array}$ \\
\hline $\begin{array}{l}\text { Total } \\
\text { XMg }\end{array}$ & $\begin{array}{r}100.68 \\
90.31\end{array}$ & $\begin{array}{l}99.93 \\
90.94\end{array}$ & $\begin{array}{l}99.74 \\
90.11\end{array}$ & $\begin{array}{r}99.66 \\
90.38\end{array}$ & $\begin{array}{r}100.38 \\
88.94\end{array}$ & $\begin{array}{l}99.29 \\
91.66\end{array}$ & $\begin{array}{l}99.70 \\
89.92\end{array}$ & $\begin{array}{r}100.55 \\
90.59\end{array}$ & $\begin{array}{l}99.95 \\
90.35\end{array}$ & $\begin{array}{l}99.64 \\
85.78\end{array}$ \\
\hline
\end{tabular}


Table 1. Continued

Representative clinopyroxenes

El Aprisco

Cerro Pelado

\begin{tabular}{|c|c|c|c|c|c|c|c|c|c|c|c|c|}
\hline Sample & $\begin{array}{c}55569 \\
\text { A30 }\end{array}$ & $\begin{array}{c}55570 \\
\text { A30 }\end{array}$ & $\begin{array}{c}72688 \\
\text { A57 }\end{array}$ & $\begin{array}{c}72689 \\
71(\mathrm{Cpx}-2)\end{array}$ & $\begin{array}{c}72689 \\
\text { A75 }\end{array}$ & $\begin{array}{c}72690 \\
\text { A42 }\end{array}$ & $\begin{array}{c}72691 \\
\text { A66 }\end{array}$ & $\begin{array}{c}65290 \\
\text { A31 }\end{array}$ & $\begin{array}{c}65298 \\
22(\mathrm{Cpx}-2)\end{array}$ & $\begin{array}{c}65298 \\
\mathrm{~A} 4\end{array}$ & $\begin{array}{c}72674 \\
101(\mathrm{Cpx}-2)\end{array}$ & $\begin{array}{c}72674 \\
\text { A96 }\end{array}$ \\
\hline $\mathrm{Si}_{2}$ & 52.29 & 51.75 & 51.89 & 49.86 & 51.05 & 52.37 & 52.57 & 51.19 & 53.03 & 51.36 & 53.97 & 49.49 \\
\hline $\mathrm{Ti}_{2}$ & 0.46 & 0.23 & 0.42 & 0.80 & 0.41 & 0.33 & 0.01 & $\bullet .54$ & 0.51 & 0.52 & 0.62 & 1.39 \\
\hline $\mathrm{Al}_{2}{ }_{3}$ & 6.90 & 5.72 & 4.87 & 8.11 & 6.07 & 7.61 & 4.44 & 7.37 & 4.43 & 7.11 & 3.12 & $6.7 \bullet$ \\
\hline $\mathrm{Fe}$ & 2.60 & 2.87 & 2.86 & 3.08 & 2.73 & 2.46 & 2.33 & 3.46 & 4.21 & 3.31 & 6.11 & 4.80 \\
\hline $\mathrm{Mn} \bullet$ & 0.05 & 0.06 & 0.14 & 0.02 & 0.10 & bdl & 0.05 & 0.09 & 0.17 & 0.06 & •.16 & bdl \\
\hline $\mathrm{MgO}$ & 14.94 & 15.81 & 15.66 & 15.65 & 15.35 & 14.44 & 16.64 & 15.72 & 17.72 & 15.66 & $17.9 \bullet$ & 14.39 \\
\hline $\mathrm{Ca}$ & 19.69 & 21.15 & 22.26 & 18.80 & 21.58 & 20.55 & 21.29 & 18.90 & 18.17 & 19.09 & 16.57 & 20.95 \\
\hline $\mathrm{Na}_{2} \bullet$ & 1.62 & 1.35 & 0.87 & 1.19 & 0.98 & 1.64 & 0.89 & $1.6 \bullet$ & 1.21 & 1.61 & 1.04 & 1.04 \\
\hline $\mathrm{K}_{2}$ & bdl & bdl & bdl & bdl & bdl & 0.01 & bdl & 0.01 & bdll & bdl & 0.03 & bdl \\
\hline $\mathrm{Ni}$ & 0.04 & 0.08 & 0.03 & 0.01 & 0.03 & 0.07 & 0.05 & bdl & bdl & 0.01 & 0.01 & 0.01 \\
\hline $\mathrm{Cr}_{2} a_{3}$ & 0.79 & 0.83 & 0.54 & 1.50 & 0.7 & 0.94 & - 61 & 0.74 & 0.97 & 0.66 & 0.63 & 0.59 \\
\hline Total & 99.36 & 99.85 & 99.55 & 99.03 & 98.99 & 100.42 & 98.88 & 99.63 & 100.41 & 99.39 & 100.14 & 99.34 \\
\hline $\mathrm{XMg}$ & 91.10 & 90.79 & 90.72 & 90.06 & 90.98 & 91.56 & 92.65 & 88.98 & 88.20 & 89.33 & 83.94 & 84.20 \\
\hline $\mathrm{XCr}$ & 0.07 & 0.09 & 0.06 & 0.08 & 0.07 & 0.07 & 0.10 & 0.09 & 0.13 & 0.06 & 0.12 & 0.09 \\
\hline
\end{tabular}

Representative spinels

El Aprisco

Cerro Pelado

\begin{tabular}{|c|c|c|c|c|c|c|c|c|c|c|c|c|}
\hline Sample & $\begin{array}{c}55569 \\
\text { A4 }\end{array}$ & $\begin{array}{c}5557 \bullet \\
\text { A16 }\end{array}$ & $\begin{array}{l}72688 \\
\text { A144 }\end{array}$ & $\begin{array}{c}72689 \\
\text { A90 }\end{array}$ & $\begin{array}{l}72690 \\
\text { A55 }\end{array}$ & $\begin{array}{c}72691 \\
121 \text { sympl. }\end{array}$ & $\begin{array}{c}72691 \\
56 \text { sympl. }\end{array}$ & $\begin{array}{c}6529 \\
\text { A29 }\end{array}$ & $\begin{array}{c}65290 \\
\text { A30 (Sp-2) }\end{array}$ & $\begin{array}{c}65298 \\
\text { A5 (Sp-2) }\end{array}$ & $\begin{array}{c}65298 \\
\text { Al }\end{array}$ & $\begin{array}{c}72674 \\
\text { A13 }\end{array}$ \\
\hline $\mathrm{Si}_{2}$ & 0.07 & 0.02 & 0.13 & 0.07 & 0.06 & 0.05 & 0.04 & 0.08 & 0.11 & 0.11 & 0.08 & $\begin{array}{l}0.11 \\
249\end{array}$ \\
\hline $\mathrm{Al}_{2} \mathbf{\Theta}_{3}$ & 56.51 & 52.23 & 55.96 & 57.78 & 58.40 & 53.85 & 51.03 & 56.97 & 53.15 & 51.64 & 56.83 & 20.76 \\
\hline $\mathrm{Cr}_{2} \bullet_{3}$ & 10.17 & 15.73 & 9.61 & 8.84 & 10.15 & 13.10 & 16.32 & 10.17 & 13.11 & 14.46 & 9.31 & 35.80 \\
\hline $\mathrm{Fe}$ & 11.97 & 12.09 & 13.23 & 12.00 & 10.57 & 10.90 & 11.73 & 12.55 & 11.99 & 11.10 & 11.15 & 26.37 \\
\hline $\mathrm{Mn} \bullet$ & 0.04 & 0.10 & $\bullet .15$ & ๑.11 & 0.041 & 0.10 & 0.07 & 0.02 & 0.11 & 0.13 & 0.10 & 0.19 \\
\hline $\mathrm{Ni} \bullet$ & 0.34 & 0.33 & 0.29 & 0.48 & 0.39 & 0.40 & •. 25 & 0.32 & 0.34 & 0.28 & 0.34 & 0.11 \\
\hline $\mathrm{Mg} \bullet$ & 20.41 & 19.65 & 19.81 & 20.49 & 21.12 & 20.82 & 19.90 & 20.23 & 20.62 & 20.93 & 21.56 & 13.23 \\
\hline $\mathrm{Ca}$ & 0.03 & 0.03 & 0.09 & 0.01 & bdl & 0.03 & 0.06 & 0.01 & 0.02 & 0.03 & 0.02 & 0.18 \\
\hline $\mathrm{Na}_{2}$ & 0.04 & 0.01 & 0.04 & 0.01 & 0.01 & bdl & bdl & bdl & bdl & bdl & 0.01 & bdl \\
\hline $\mathrm{K}_{2} \bullet$ & bdl & bdl & bdl & bdl & bdl & bdl & bdl & 0.01 & bdl & bdl & 0.02 & bdl \\
\hline Total & 100.00 & 100.28 & 99.74 & 100.12 & 100.84 & 99.54 & 99.65 & 100.78 & 100.13 & 99.36 & 99.93 & 99.22 \\
\hline $\mathrm{XCr}$ & 10.78 & 16.81 & 10.33 & 9.30 & 10.43 & 14.05 & 17.66 & 10.71 & 14.21 & 15.83 & 9.90 & 53.64 \\
\hline $\mathrm{XMg}$ & $\bullet .75$ & 0.74 & 0.73 & 0.75 & 0.78 & $\bullet .77$ & $\bullet .75$ & 0.74 & 0.76 & 0.77 & 0.78 & 0.48 \\
\hline
\end{tabular}


Representative amphiboles and phlogopites

\begin{tabular}{|c|c|c|c|c|c|c|c|c|c|}
\hline \multirow[b]{2}{*}{ Sample } & \multicolumn{6}{|c|}{ Amphibole } & \multicolumn{3}{|c|}{ Phlogopite } \\
\hline & $\begin{array}{c}55569 \\
\text { A27 }\end{array}$ & $\begin{array}{c}55570 \\
\text { A35 }\end{array}$ & $\begin{array}{c}72689 \\
\text { A85 }\end{array}$ & $\begin{array}{c}72691 \\
\text { A55 }\end{array}$ & $\begin{array}{c}72688 \\
\text { A51 }\end{array}$ & $\begin{array}{c}72688 \\
\text { A68 }\end{array}$ & $\begin{array}{c}65298 \\
\text { A13 }\end{array}$ & $\begin{array}{c}65298 \\
\text { A19 }\end{array}$ & $\begin{array}{l}72674 \\
\text { A116 }\end{array}$ \\
\hline $\mathrm{Si}_{2}$ & 43.83 & 42.65 & 42.79 & 43.63 & 42.19 & 42.59 & 37.64 & 38.13 & 35.98 \\
\hline $\mathrm{TiO}_{2}$ & 1.48 & 0.58 & 0.73 & 0.42 & 1.33 & 1.33 & 3.10 & 3.12 & 6.76 \\
\hline $\mathrm{Al}_{2} \boldsymbol{\bullet}_{3}$ & 14.25 & 15.43 & 15.05 & 14.97 & 15.18 & 15.09 & 17.90 & 18.18 & 15.70 \\
\hline $\mathrm{Fe}$ & 3.72 & 3.82 & 4.04 & 3.72 & 4.40 & 4.16 & 4.17 & 3.88 & 8.08 \\
\hline $\mathrm{Mn} \bullet$ & 0.04 & 0.14 & 0.05 & 0.09 & $\bullet .12$ & 0.04 & 0.05 & bdl & 0.09 \\
\hline $\mathrm{Mg}$ & 17.22 & 17.86 & 18.09 & 18.64 & 17.67 & 17.54 & 21.35 & 20.54 & 17.66 \\
\hline $\mathrm{Ca}$ & 11.48 & 10.74 & 11.17 & 10.83 & 11.76 & 11.69 & 0.02 & 0.05 & 0.10 \\
\hline $\mathrm{Na}_{2} \bullet$ & 3.51 & 3.75 & 3.59 & 3.62 & 3.50 & 3.41 & 0.79 & $\bullet .76$ & 0.81 \\
\hline $\mathrm{K}_{2}$ & 0.02 & 0.20 & bdl & •.11 & 0.06 & 0.07 & 9.15 & 9.37 & 8.91 \\
\hline $\mathrm{Ni}$ & .25 & 0.10 & 0.19 & 0.08 & 0.07 & 0.04 & bdl & bdl & bdl \\
\hline $\mathrm{Cr}_{2} \Theta_{3}$ & 0.57 & 1.39 & 0.72 & 1.34 & 0.73 & 0.77 & 0.78 & 0.81 & 0.16 \\
\hline Total & 95.54 & 95.17 & 95.50 & 96.02 & 96.20 & 95.92 & 94.93 & 94.83 & 94.25 \\
\hline $\mathrm{XMg}$ & 89.18 & 89.29 & 88.87 & 89.93 & 87.75 & 88.27 & 90.14 & 90.43 & 79.58 \\
\hline $\mathrm{XCr}$ & 2.64 & 5.71 & 3.10 & 5.66 & 3.14 & 3.30 & 2.85 & 2.90 & 0.69 \\
\hline
\end{tabular}

Interstitial glasses

El Aprisco

Cerro Pelado

\begin{tabular}{|c|c|c|c|c|c|c|c|c|}
\hline Sample & $\begin{array}{c}72689 \\
\text { A22 }\end{array}$ & $\begin{array}{c}72689 \\
\text { A24 }\end{array}$ & $\begin{array}{c}65290 \\
\text { A34 }\end{array}$ & $\begin{array}{c}65290 \\
\text { A37 }\end{array}$ & $\begin{array}{c}72674 \\
\text { A7 }\end{array}$ & $\begin{array}{c}72674 \\
\mathrm{Al}\end{array}$ & $\begin{array}{c}72674 \\
\text { A5 }\end{array}$ & $\begin{array}{c}72674 \\
\text { A109 }\end{array}$ \\
\hline $\begin{array}{l}\mathrm{Si} 2 \\
\mathrm{Ti} \\
\mathrm{Al}_{2} \\
\mathrm{Fe} \\
\mathrm{Mn} \\
\mathrm{Mg} \\
\mathrm{Ma} \\
\mathrm{Ca} \\
\mathrm{Na}_{2} \\
\mathrm{~K}_{2} \\
\mathbf{P}_{2} \\
\mathrm{Cr}_{2}\end{array}$ & $\begin{array}{c}57.73 \\
0.84 \\
22.22 \\
2.01 \\
\text { bdl } \\
3.09 \\
6.70 \\
5.54 \\
0.06 \\
0.11 \\
0.06\end{array}$ & $\begin{array}{r}56.79 \\
0.80 \\
21.87 \\
2.25 \\
\mathbf{0 . 0 3} \\
3.48 \\
7.07 \\
5.02 \\
0.03 \\
0.08 \\
0.12\end{array}$ & $\begin{array}{r}55.66 \\
1.02 \\
21.51 \\
2.35 \\
\mathbf{0 . 0 3} \\
3.37 \\
4.77 \\
4.36 \\
4.33 \\
\mathbf{0 . 2 3} \\
\mathbf{0 . 3 7}\end{array}$ & $\begin{array}{r}54.60 \\
1.08 \\
21.63 \\
1.86 \\
0.03 \\
2.74 \\
4.81 \\
5.07 \\
4.87 \\
0.20 \\
0.37\end{array}$ & $\begin{array}{r}53.29 \\
1.92 \\
18.87 \\
5.17 \\
\mathbf{0 . 0 8} \\
2.25 \\
4.19 \\
6.26 \\
5.27 \\
\mathbf{0 . 3 5} \\
\mathbf{0 . 0 7}\end{array}$ & $\begin{array}{r}53.68 \\
2.36 \\
18.99 \\
4.63 \\
0.12 \\
1.89 \\
3.90 \\
6.06 \\
5.18 \\
0.29 \\
0.04\end{array}$ & $\begin{array}{r}58.17 \\
1.49 \\
16.52 \\
3.75 \\
\mathbf{0 . 0 2} \\
1.87 \\
2.37 \\
5.19 \\
5.44 \\
\mathbf{0 . 2 4} \\
\mathbf{0 . 0 3}\end{array}$ & $\begin{array}{r}59.74 \\
1.78 \\
16.69 \\
3.43 \\
\mathbf{0 . 0 7} \\
1.82 \\
2.30 \\
3.34 \\
5.17 \\
0.20 \\
0.03\end{array}$ \\
\hline $\begin{array}{l}\text { Total } \\
\text { XMg }\end{array}$ & $\begin{array}{r}98.42 \\
0.73\end{array}$ & $\begin{array}{r}97.34 \\
\mathbf{0 . 7 3}\end{array}$ & $\begin{array}{r}98.00 \\
0.72\end{array}$ & $\begin{array}{r}97.24 \\
\mathbf{0 . 7 2}\end{array}$ & $\begin{array}{r}97.77 \\
\mathbf{0} .44\end{array}$ & $\begin{array}{r}97.18 \\
0.42\end{array}$ & $\begin{array}{r}95.10 \\
\mathbf{0 . 4 7}\end{array}$ & $\begin{array}{r}94.56 \\
\mathbf{0 . 4 9}\end{array}$ \\
\hline
\end{tabular}

Abbreviation: bdl, below detection limits. 

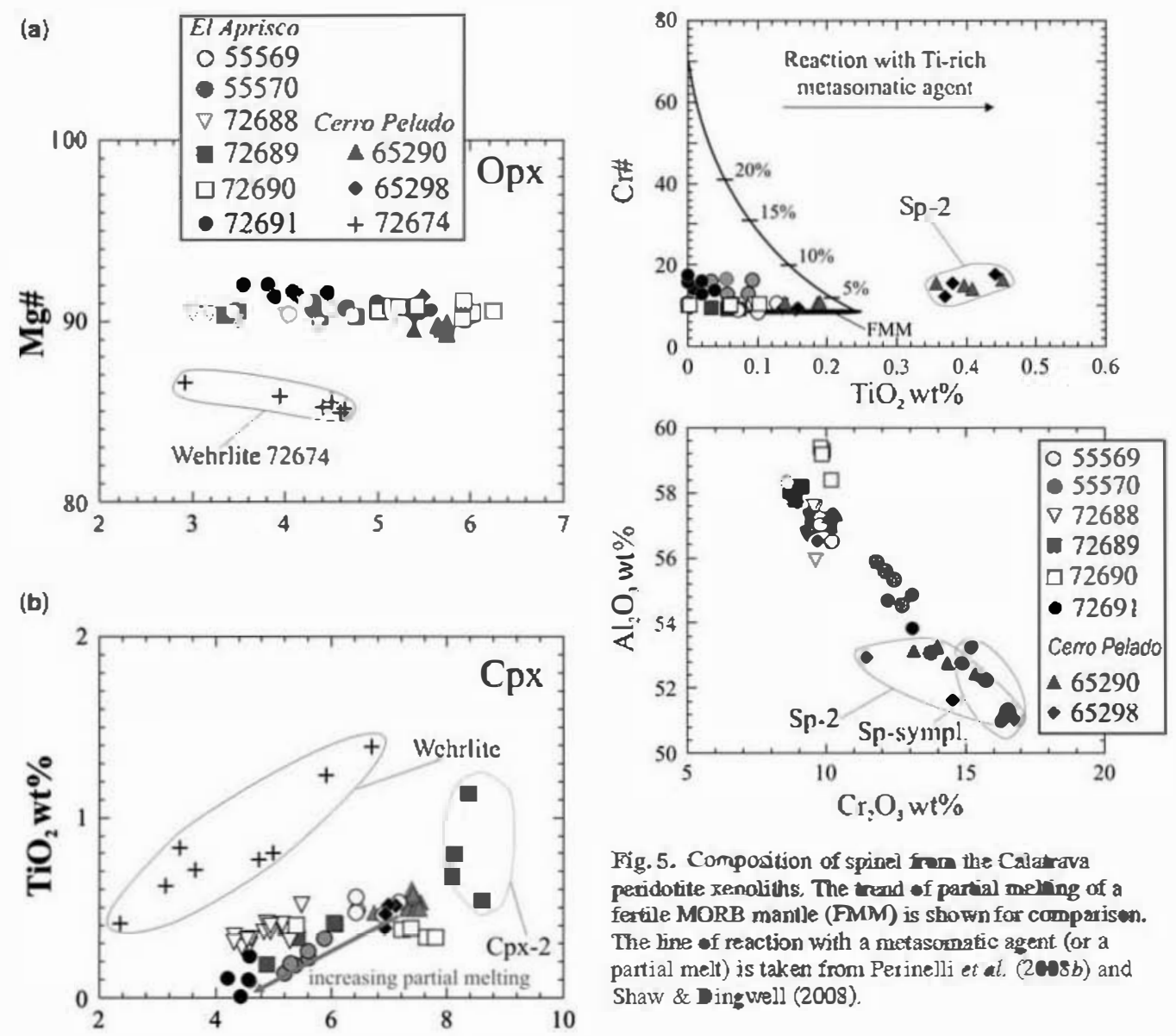

Fig. 5. Composition of spinel fon the Calatrava peridotite xenoliths. The trend of partial meling of a ferile MORB mantle (FMM) is shown for comparisen. The bine of reaction with a metasomatic agent (or a partial melt) is taken from Perinelli et al. (2cosb) and Shaw \& Dingwell (2008).

(c)

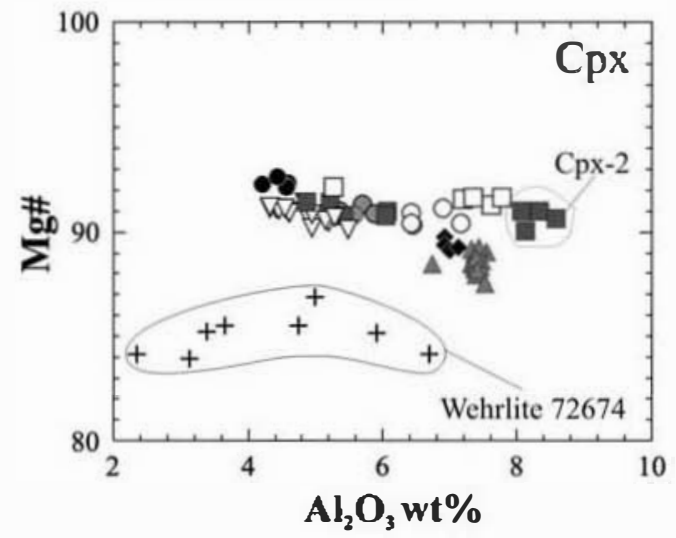

Fig. 4. Majer element compositions of pyroxenes in Calatrava peridotite xenoliths. Mg\# is llie $\mathrm{Mg}$-number. The grey arrow in (b) shwws clinopyroxeme compositional variation with a pregressive increase in peridotite partial meling (Uysal et al. 2007).

this extreme composition they have not been plotted in Figure 5.

The low $\mathrm{Cr}$-number of primary spinels from the Calatrava herzolite xenoliths combined with the areraged $\mathrm{Mg}$-number of the coesisting olivine make them plot in the OSMA (olivine-spinel martle array) closer to fertile mantle compositions (Arai 1992) than other lherzolite xenolith suites from Iberia (Fig. 6). The Cr-spinel of wehrlite 72674 pl ots outside the OSMA, further to the tight of the Olot harzburgite field (Fi . 6).

Amphibele is a typical interstitial minerall ecally sutrounding primary spinel in the therz lite samples from the El Aprisco maar. Its composition is mainly pargasite. Mg-numbers range from 86.9 to 91.2, and are positively correlated with $\mathrm{Cr}_{2} \mathrm{O}_{3}$ content $(0.7-1.4 w t \%)$ but inversely with $\mathrm{TiO}_{2}(1.48-$ .69 wit\%) and $\mathrm{CaO}(11.9-11.0$ wit\%) content. Amphibole in lherzolites 72691 and 55570 shows the highest $\mathrm{Mg}$ - and $\mathrm{Cr}$-mminhers (89.3-91.2 and 4.8-5.7, respectively), and the lowest $\mathrm{Ti}_{2}$ and $\mathrm{Ca}$ content $(0.3-0.6$ and 10.4-10.8 wrt\%, 


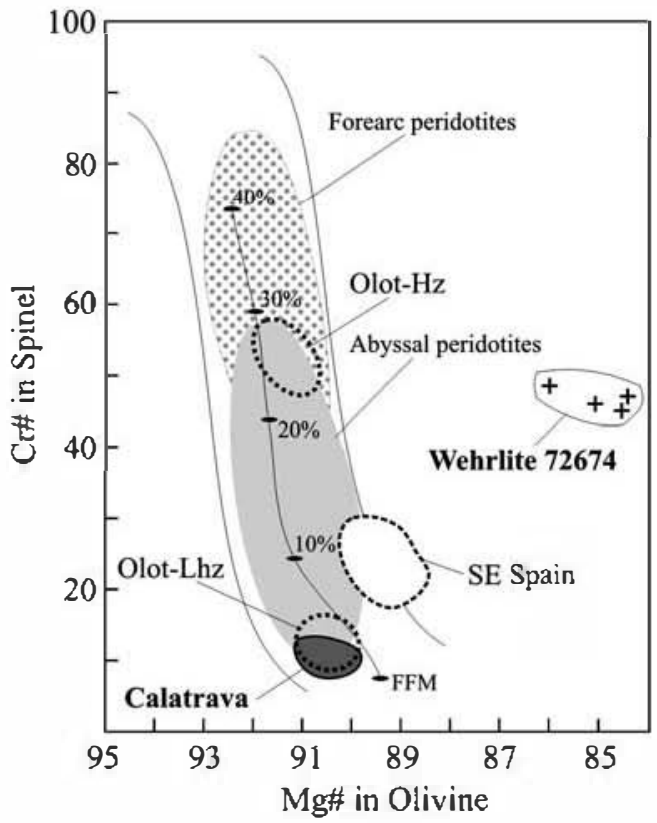

Fig. 6. Cr-number $(1 \omega \times \mathrm{Cr} /(\mathrm{Cr}+\mathrm{Al})$ in spinel $v$. Mg-number in coexisting livine for loerian mantle xen liths. Mineral data from the Olıtv $\bullet$ lcanic field ( $\mathrm{Lhz}$, lherzelites; $\mathrm{Hz}$, harzburgites) are taken from Bianchini et al. (2007) and Galán et al. (2058). Data from the SE Spain volcanic field (mainly from the Tallante volcanic centre) are from Beccaluva et al. (2004) and Shimizu et al. (2N4). OSMA-livine-spinel mantle array (A rai 1994). FMM: fertile MORB mantle and partial meling trends are from Arai (1992). Fields forearc and abyssal peridetites are from Pearce et al. (2000).

respectively). The low-K content of Calatrava amphib les $(<0.11 \mathrm{w} \%)$ is remarkable compared to other mantle xenolith suites (Coltorti et al. 2007a), except these from some lot lherzolites (OLT-21 and OLT-23 samples: Bianchini et al. 2007).

Phlogepite is only found in mantle xenoliths from the Cerro Pelade centre with Mg-number (90.2-90.4) similat to coexisting clinopytoxene but with higher $\mathrm{Cr}$-number (2.8-2.9) (Table 1). Phlogopite included in clinopyroxene from wehrlite 72674 is markedly peorer in $\mathrm{Cr}$ and $\mathrm{Mg}$ values ( $\mathbf{0 . 7}$ and 79.6, respectively) but richer in $\mathrm{Ti}_{2}$ content (6.8 wt\%), similar in composition to phlogepites from associated limmerite xenoliths (anal. 6 in table 32 of Ancochea \& Nix 0 1987).

Glass veins and interstial lasses have been -bserved in some Calatrava mantle xenoliths. In xenoliths from the Cerr Pelad centre the glasses are alkaline in composition and they plot mostly in the field of rachy-andesite in the total alkalissilica (TAS) diagram (Fi. 7). However, interstitial
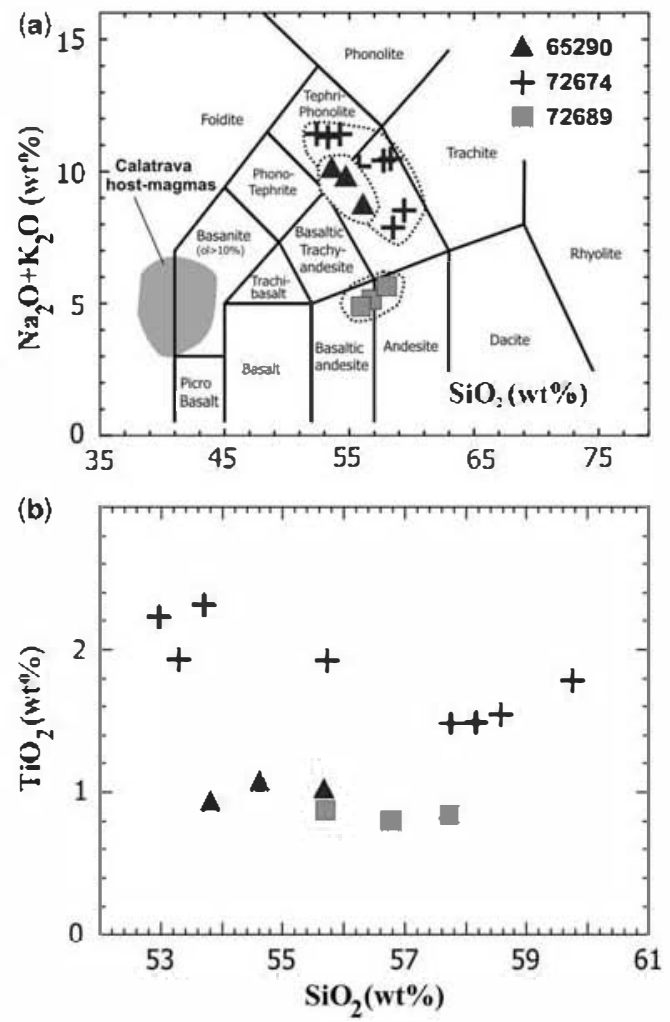

Fig. 7. Chemistry of glasses from Calatrava peridorite xenoliths. (a) The total alkali-silica (ГAS) diagram of Le Bas et al. (1986) for glasses in Calarava mantle xen liths. The grey field for Calatrava velcanic rocks is taken from Ancechea (2004). (b) $\mathrm{TiO}_{2}$-silica (in wt\%) diagram.

lass from therzelite 72689 from the El Aprisce volcan is subalkaline in compesition owing to its low $K_{2} \bullet$ content (Table 1), and plots mostly in the field of basalic andesite in the TAS diagram. The Calatrava glasses are compositionally heterogeneous, and thus, the high $\mathrm{TiO}_{2}$ and $\mathrm{FeO}$ (and low $\mathrm{Al}_{2} \mathrm{O}_{3}$ and $\mathrm{Mg}$ ) content shown by lasses from wehrlite $72674(1.8-2.3$ and $3.3-5.8 \mathrm{wt} \%$, respectively) (Table 1) ar e quite remarkable (Fi . 7).

\section{Pressure-temperature $\mathbf{P}-\mathrm{T}$ estimates}

The lithospheric thickness in central Spain seems to be almost constant over a large area at around $110 \mathrm{~km}$, while the depth to the Meh is around $32 \pm 2 \mathrm{~km}$ (Fernàndez et al. 1998). The depth of xenolith extraction is constrained to less than $70 \mathrm{~km}$ (c. $20 \mathrm{kbar}$ ) by the absence of garnet in the Therzolite suite. Although for spinel peridotites there are no precise geobarometers (e.g. Pearson et al. 2005; Ackerman et al. 2007), we have used 
Table 2. $\mathbf{P}-\mathrm{T}$ estimates on the Calatrava mantle xenoliths

\begin{tabular}{|c|c|c|c|c|}
\hline & \multicolumn{3}{|c|}{$T\left({ }^{\bullet} \mathrm{C}\right)$} & \multirow{2}{*}{$\frac{P \text { (kbar) }}{P_{\mathrm{NU}}}$} \\
\hline & $T_{\text {WBB }}$ & $T_{\mathrm{BM}}$ & $T_{\mathbf{B K}}$ & \\
\hline \multicolumn{5}{|l|}{ El Aprisco xenoliths } \\
\hline 55569 срх30орх36 & 1093 & 1185 & 1052 & 14.6 \\
\hline 55570 срх30орх32 & 1116 & 122 & 1073 & 9.7 \\
\hline 72688 срх57op $\times 59$ & 1003 & 1036 & 866 & 6.4 \\
\hline 72689 срх75op $\times 76$ & 1054 & 1109 & 938 & 8.9 \\
\hline$(\mathrm{cpx}-2) 71$ opx 76 & 1182 & 1345 & 1163 & 13.9 \\
\hline 72690 срх42op 441 & 1079 & 1062 & 980 & 15.0 \\
\hline 72691 срх66орх65 & 1115 & 1274 & 994 & 7.6 \\
\hline \multicolumn{5}{|l|}{ Cerro Pelado xenoliths } \\
\hline 65290 срх31 ор $\times 27$ & 1149 & 1382 & 1112 & 13.4 \\
\hline 65298 срх4орх3 & 1165 & 1321 & 1123 & 13.8 \\
\hline$(\mathrm{cpx}-2) 22$ op 23 & 1255 & 1420 & 1209 & 7.0 \\
\hline 72674 срх96ор x108 & 1056 & 1050 & 986 & 7.6 \\
\hline$(\mathrm{cpx}-2) 1010 \mathrm{l} x 110$ & 1243 & 1500 & 1207 & 7.0 \\
\hline
\end{tabular}

the estimations of Nimis \& Ulmer (1998) for clin॰pyroxene in order to approximate the depth of extraction of the lherzolite xenoliths (Table 2). Pressure estimates range from $14-15 \mathrm{kbar}$, for weakly metas matized lherzelites (e.g. anydrøus lherzolite 72690 , Table 2), to 7-8 kbar for the most depleted lherz@lites (72691) or wehrlite varieties (72674). These low-pressure values are unrealistic for mantle depths but suggest that xenoliths could have been trapped from a shallow lithospheric mantle close to Møh॰ depths. The second generation -f clinøpyrøxene, related either t॰ glass veining

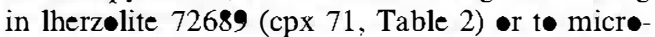
aggregates around spinel in lherzelite 65298 (cpx 22, Table 2), yields $P$ estimates mostly $\bullet$ verlapping those from primary clinøpyrøxene (13.9$7.0 \mathrm{kbar})$. These unrealistic low-pressure estimates are als in disagreement with the absence of replacement of spinel by plagioclase in the studied samples.

Temperatures were calculated using three calibrations of the tw-pyroxene geothermometer (Wood \& Bann• 1973; Bertrand \& Mercier 1985; Brey \& Köhler 1990). In general there is a goed agreement between the different methods used, although Bertrand \& Mercier's (1985) temperatures are slightly higher than the tw - other estimates (Table 2). Most lherzelites yield $T$ estimates in the range of $1000-1165^{\circ} \mathrm{C}$, whereas neof $\bullet$ rmed clin॰pyroxene give a still higher temperature range of $1180^{\circ}-1255^{\circ} \mathrm{C}$. We have to bear in mind that most

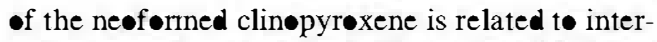
stitial glass (e.g. cpx 71 in lherzelite 72689 and cpx 101 in wehrlite 72674, Table 2). Thus, geothermømetry suggests søme kind of reheating at depth to explain the therrnal increase $\left(c .100^{\circ} \mathrm{C}\right)$. The xenelith reaction with high-temperature magmas (e.g. silica-undersaturated alkaline magmas have liquidus temperatures of arøund $1250-1300^{\circ} \mathrm{C}$ : Perinelli et al. 2008 a) may explain the higher temperature of the second-generation clinøpyrøxene in the Calatrava peridetites.

\section{Trace element mineral composition}

Trace element analyses of representative main minerals from tw॰ lherzelites are reported in Table 3. REE primitive mantle-normalized patterns

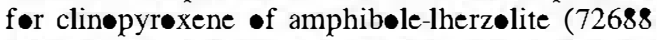
from the E1 Aprisce centre) are LREE-enriched $\left(\mathrm{La}_{\mathrm{N}} / \mathrm{Sm}_{\mathrm{N}}=10.4-13.7\right)$ and almest flat in HREE (Fig. 8). The coexisting orthopyroxene displays a similar trend in some LREE patterns (although showing an inflection to Nd instead of $\mathrm{Sm}$ as in cpx patterns) but are positively fractionated in HREE (Fig. 8). Olivine shows a similar REE

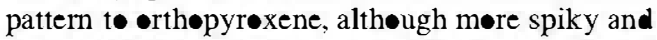
less HREE enriched (n๑t sh॰wn, Table 3). Similarly, multitrace element patterns nornalized to primitive mantle for clinøpyroxene show a marked $\mathrm{Th}-\mathrm{U}$ enrichment, and $\mathrm{Nb}-\mathrm{Ta}$ and, to a lesser extent, $\mathrm{Zr}-\mathrm{Ti}$ negative anomalies (Fig. 8). Coexisting -rthopyroxene and, less markedly, elivine show similar trace element patterns.

Amphibøle REE patterns perfectly mimic those -f the cœexisting clinopyroxene (Fig. 8). Inc®mpatible element patterns are alsø similar to these of clinøpyrexene but with higher LILE (large iøn lithophile elements) and $\mathrm{Ti}$ content. The high $\mathrm{Th}-\mathrm{U}$ content of amphibøle and assøciated clin-pyroxene suggests an enrichment process related to melt instead of a volatile-rich fluid as the metasmatizing agent (see als• Coltorti et al. 2007b). This marked $\mathrm{Th}-\mathrm{U}$ positive anomaly highlights the preminent $\mathrm{Nb}$-Ta negative an॰maly of the El Aprisc• 
Table 3. Trace element composition of minerals from the Calatrava Iherzolite xenoliths

\begin{tabular}{|c|c|c|c|c|c|c|c|c|c|}
\hline & \multicolumn{6}{|c|}{72688 (El Aprisco) } & \multicolumn{3}{|c|}{65290 (Cerro Pelado) } \\
\hline & $\begin{array}{c}\mathrm{ol} \\
(n=4)\end{array}$ & $\begin{array}{c}\text { cpx } \\
(n=12)\end{array}$ & $\begin{array}{c}\text { cpx-2 } \\
(n=3)\end{array}$ & $\begin{array}{c}\text { opx } \\
(n=4)\end{array}$ & $\begin{array}{c}\text { amph } \\
(n=21)\end{array}$ & $\stackrel{s p}{(n=2)}$ & $\begin{array}{c}\text { ol } \\
(n=9)\end{array}$ & $\begin{array}{c}\text { cpx } \\
(n=24)\end{array}$ & $\begin{array}{c}\text { opx } \\
(n=12)\end{array}$ \\
\hline $\mathrm{Sc}$ & 6.783 & 77.70 & 76.43 & 23.88 & 66.59 & 0.279 & 6.542 & 62.83 & 27.89 \\
\hline V & 16.62 & 202 & 199 & 86.83 & 331 & 351 & 5.470 & 187 & 98.93 \\
\hline $\mathrm{Cr}$ & 457 & 3803 & 4117 & 1840 & 4568 & 47750 & 128 & 4235 & 2868 \\
\hline Co & 116 & 18.70 & 19.00 & 54.35 & 32.87 & 187 & 139 & 19.44 & 57.45 \\
\hline $\mathrm{Zn}$ & 37.88 & 14.60 & 15.26 & 28.38 & 11.46 & 885 & 78.99 & $11.7 \bullet$ & 46.71 \\
\hline $\mathrm{Rb}$ & 0.059 & 0.048 & $\bullet .051$ & 0.053 & 3.377 & 0.084 & 0.064 & 0.048 & 0.052 \\
\hline $\mathrm{Sr}$ & 0.180 & 172 & 168 & 0.935 & 529 & 0.054 & 0.060 & 146 & 0.803 \\
\hline Y & •.214 & 18.63 & 18.467 & 1.137 & 33.629 & 0.023 & $\bullet .113$ & 24.26 & 2.32 \\
\hline $\mathrm{Zr}$ & 0.138 & 11.36 & 11.30 & •.632 & 12.13 & 0.074 & 0.227 & 119 & 12.94 \\
\hline $\mathrm{Nb}$ & $\bullet .017$ & $\bullet .014$ & $\mathbf{0 . 0 1 5}$ & 0.014 & 0.154 & 0.094 & 0.025 & 1.118 & 0.082 \\
\hline $\mathrm{Ba}$ & 0.281 & 2.862 & 0.189 & •.142 & 845 & 0.084 & 0.142 & 0.089 & 0.086 \\
\hline $\mathrm{Hf}$ & 0.068 & 0.758 & $\bullet .717$ & $\bullet .052$ & 0.798 & 0.076 & 0.039 & 3.585 & 0.332 \\
\hline $\mathrm{Ta}$ & 0.012 & $\bullet .011$ & 0.009 & 0.014 & 0.010 & 0.025 & 0.012 & - 472 & 0.023 \\
\hline $\mathbf{P b}$ & 0.050 & 1.311 & 1.357 & 0.057 & 5.543 & 0.056 & 0.045 & $\mathbf{0 . 0 5 1}$ & 0.037 \\
\hline Th & 0.080 & 4.115 & 4.020 & $\bullet .253$ & 5.356 & 0.028 & 0.014 & 0.108 & 0.012 \\
\hline U & 0.018 & $1.2 \bullet 3$ & 1.183 & 0.052 & 1.467 & 0.016 & 0.009 & 0.015 & 0.007 \\
\hline $\mathrm{La}$ & 0.047 & 27.60 & 27.10 & •.195 & 40.63 & $\mathbf{0 . 0 2 1}$ & 0.013 & 5.399 & 0.024 \\
\hline $\mathrm{Ce}$ & 0.044 & 31.31 & 30.57 & 0.218 & 44.92 & 0.017 & 0.013 & 17.18 & $\bullet .136$ \\
\hline Pr & 0.011 & 2.278 & 2.11 & 0.018 & 3.276 & 0.008 & 0.006 & 3.448 & 0.034 \\
\hline $\mathrm{Nd}$ & 0.084 & 6.235 & 5.527 & 0.107 & 9.175 & 0.104 & 0.090 & 19.53 & 0.244 \\
\hline $\mathrm{Sm}$ & 0.062 & 1.458 & 1.313 & 0.071 & 2.220 & 0.060 & 0.058 & 5.391 & $\bullet .139$ \\
\hline $\mathrm{Eu}$ & 0.019 & e.556 & 0.558 & 0.024 & 0.909 & 0.018 & 0.015 & 1.637 & 0.046 \\
\hline Gd & 0.068 & 2.378 & $2.46 \bullet$ & 0.104 & 4.124 & 0.107 & 0.062 & 5.575 & 0.201 \\
\hline $\mathrm{Tb}$ & 0.009 & 0.433 & 0.447 & $\bullet .014$ & 0.746 & 0.013 & 0.007 & 0.814 & 0.040 \\
\hline Dy & 0.052 & 3.391 & 3.22 & $\bullet .132$ & 5.710 & 0.059 & 0.049 & 5.101 & 0.343 \\
\hline Ho & 0.018 & 0.729 & 0.730 & 0.038 & 1.277 & 0.015 & 0.011 & 0.967 & 0.090 \\
\hline $\mathrm{Er}$ & 0.050 & 2.250 & 2.093 & $\bullet .159$ & 3.794 & $\mathbf{0 . 0 5 1}$ & 0.048 & 2.542 & 0.32 \\
\hline $\mathrm{Tm}$ & 0.013 & 0.319 & 0.308 & 0.028 & •.552 & 0.011 & 0.010 & $\bullet .355$ & 0.054 \\
\hline $\mathrm{Yb}$ & 0.12 & 2.198 & 2.080 & 0.302 & 3.666 & $\mathbf{0 . 0 7 5}$ & 0.067 & 2.148 & - .446 \\
\hline $\mathrm{Lu}$ & 0.018 & 0.308 & 0.294 & 0.065 & 0.545 & 0.013 & $\boldsymbol{0 . 0 1 2}$ & 0.312 & 0.075 \\
\hline Sum LREE & 0.25 & 68.88 & 66.62 & e.61 & 100.22 & •.21 & 0.18 & 50.95 & 0.58 \\
\hline Sum HREE & 0.35 & 12.01 & 11.63 & 0.84 & 20.42 & 0.35 & 0.27 & 17.81 & 1.57 \\
\hline Sum REE & 0.62 & 81.44 & 78.80 & 1.47 & 121.55 & 0.58 & 0.46 & 70.40 & 2.19 \\
\hline
\end{tabular}

Analysed phases are primary crystals except those marked as type-2 (cpx-2 of 72688 sample).

amphibele that is higher than those described in peridotite xenoliths, with amphibeles generated in the mantle wedge abøve subduction zønes (suprasubduction amphibøles of Coltorti et al. 2007a).

Clinøpyrexene in lherzelite $65290 \mathrm{fr} \bullet \mathrm{m}$ the Cerr• Peladı maar displays completely different REE and trace element patterns to the previous lherzølite. It shøws a peculiar upwards-cœnvex shape -f LREE patterns (with the peak located at Nd) combined with low HREE content $\left(\mathrm{Lu}_{\mathrm{N}}\right.$ down to $9 \times \mathrm{Ch}$ ). Incompatible element-normalized patterns sh॰w a pøsitive fractionation up t॰ the REE where the pattern becomes almost flat, except for a small Ti negative anømaly (Fig. 8). Orthøpyrøxene (and -livine) does n๑t mimic the REE patterns of coexisting clinopyroxene, instead shøwing a marked positive fractionation in the REE profiles. Incompatible element patterns are more spiky than those of the coexisting clinopyroxene but fluctuating close to $0.1-1 \times \mathrm{Ch}$ values (Fig. 8). A positive $\mathrm{Zr}-\mathrm{Hf}$

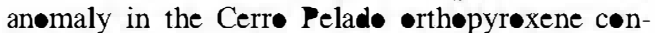
trasts with the small negative $\bullet$ ne in El Aprisce.

Similar LREE-Th-U-enriched clinøpyrøxenes to these of lherzolite 72688 have been described in the Olot lherzelite xenolith suite (Bianchini et al. 2007; Galán et al. 2008), but the upwardsconvex LREE shape has been only described in -lot harzburgites, althøugh displaying a more severe HREE-Zr-Ti depletion (Bianchini et al. 2007). Fe-rich lherzelites from the northermost El

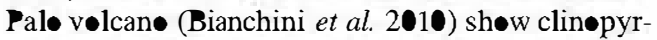
-xene REE patterns similar to these frøm the Cerr Pelade lherzelite 65290, althøugh with lower total REE content and less prominent convex shape at the MREE. Moreover, clinopyroxene from

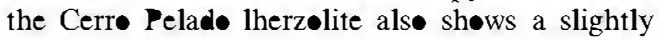



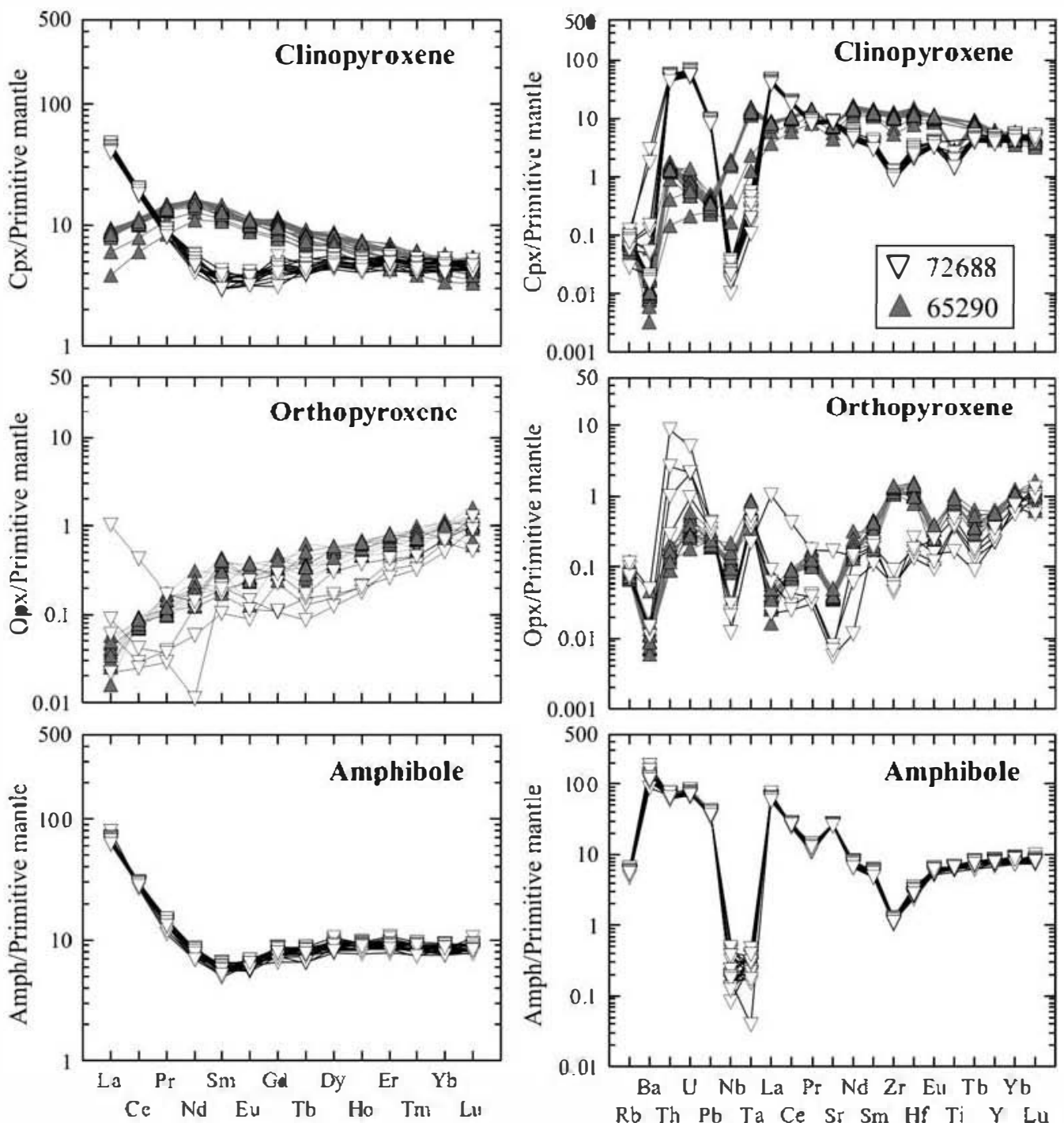

Fig.8. Primerdial mantle-normalized REE and race element patterns for minerals in the Calattava periclotite xenoliths. Nornalizing values from McDonough \& Sun (1995).

hisher $\mathrm{Zr}-\mathrm{Hr}-\mathrm{Ta}-\mathrm{Nb})-\mathrm{Y}$ content than these from El Pale. The race element content of clinepyroxenes from the SE Spain peridotite xenoliths (Beccaluva et al. 2004) are completely different t• those of lberian alkaline volcanic fields.

\section{Whole-rock composition}

\section{Major and trace elements}

Whole-tock majer and trace element composition of Calatrava mantle xenoliths are reported in Table 4. They are close to primordial mantle composition -r moderately depleted, but their lower $\mathrm{Ni}, \mathrm{Cr}$ and Mg content when compared to other Spanish mantle xenolith suites (Fir. 9) suggests a more fertle lithospheric mantle composition beneath central Spain. In most samples the $\mathrm{Al}_{2} \mathbf{O}_{3}, \mathrm{Ca}$, $\mathrm{FeO}$ and $\mathrm{Ti}_{2}$ content exibits negative correlations with $\mathrm{Mg}$ (Fig. 9), which is a commen feature in mantle xenolith suites (e.t. Downes 2001; Ackerman et al. 2007; ianchini et al. 2007). This is atributed to depletion during partial melting of the lithospheric mantle (Niu 1997). The most clinopyroxene-rich samples of Calatrava xenoliths (including wehrlite 72674) define a change in the 
slope of this compositional trend or an •pposite evolution (e.g. positive $\mathrm{Al}_{2} \mathbf{O}_{3}$ and $\mathrm{Si}_{2}$ correlations with $\mathrm{Mg}$ ) (Fig. 9). The wehrlite sample (72674) shows the most extreme composition of this trend (the highest $\mathrm{FeO}$ and $\mathrm{TiO}_{2}$, and the lowest $\mathrm{SiO}_{2}$, $\mathrm{Al}_{2} \boldsymbol{O}_{3}$ and $\mathrm{Cr}-\mathrm{Ni}$, content).

Most of the studied Calatrava lherzolites have high Mg-numbers (0.91-0.92), which are close to primordial mantle estimates (Table 4). Høwever, lower $\mathrm{Mg}$-numbers were determined for wehrlite (0.87, Table 4) and the Ti-Fe-rich

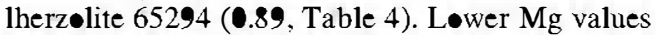
have been found in $\bullet$ ther Calatrava lherzelites (see Bianchini et al. 2010), which als• shew a higher Fe-rich character than $\bullet$ ur studied xenøliths (Fig. 9).

Whøle-røck REE patterns are varied in Calatrava

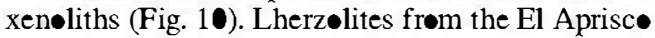
centre show a wide compesitional trend varying from LREE-enriched steep patterns (72691 sample), through the downwards-convex shape of LREE patterns (72688 sample), identical to these -f its correspønding clinopyrøxene (see alsø Fig. 8), towards the slightly negative REE patterns (although slightly upwards-convex in the LREE) (sample 55569) (Fig. 10a). The tw samples with lower LREE content are either anhydrous (72690) -r contain very minør modal amøunts of amphibole (55569). They have similar trace element patterns to the least refractory Olot lherzolites (Beccaluva et al. 2004).

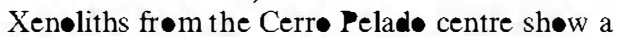
møre hømøgeneøus shape of REE patterns frøm a LREE-enriched (e.g. wehrlite 72674) to a slightly upwards-convex LREE pattern, similar to its corresp॰nding clinøpyroxene (sample 65290) (Fig. 10b). As for the major elements, lherzelites from the El Pale centre (Bianchini et al. 2010) show REE patterns more similar to those of the Cerrø Pelad• lherzolites. Mest of the LREE-enriched patterns are attributed to cryptic metas matism, their variability related both to the nature of the metas matic agent and the efficiency of the metas matic process (e.g. Bianchini et al. 2007).

In addition to LREE, studied Calatrava xenøliths are alsø enriched in LII.E, Th and U (Fig. 10), although there is søme scattering in the data owing to their low trace element concentrations, sømetimes below analytical detection limits (Table 4). By contrast, the less LREE-enriched samples show

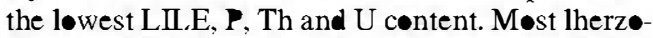
lite xenøliths from the El Aprisc• centre display $\mathrm{Nb}-\mathrm{Ta}(\mathrm{Zr}-\mathrm{Hf}$ and $\mathrm{Ti}$ ) negative anımalies in trace element patterns (Fig. 10c).

\section{Sr-Nd isotopes}

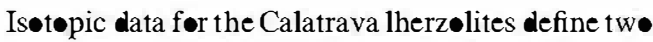
-verlapping compositional fields, depending $\bullet$ the sampled v•lcanic centre. El Aprisc have slightly more radiegenic $\mathrm{Sr}\left({ }^{87} \mathrm{Sr} /{ }^{86} \mathrm{Sr}\right.$ ratios 0.7035-0.7044) than the Cerr• Pelado samples $\left({ }^{87} \mathrm{Sr} /{ }^{86} \mathrm{Sr}\right.$ ratios $0.7032-\mathbf{0 . 7 0 3 7}$ ) (Table 5)

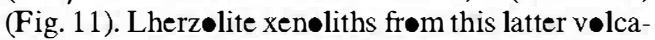
nic centre shew a compesition mere similar to the host magmas than xenøliths from El Aprisc•

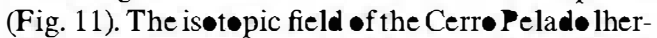
zolites includes the compositional field defined

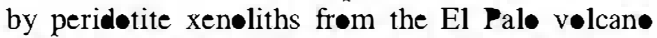
(Bianchini et al. 2010). The whole lherzelite is $\bullet$ pic field defines a wider compositional field than that $\bullet$ their host magmas. This suggests a slightly more enriched character of the Calatrava xeneliths when compared to lithospheric-asthenespheric mantle søurces of the host alkaline ultrabasic vølcanic magmas. This enriched is topic signature is remarkable when compared to mantle xenoliths frøm the other Iberian Cenøz•ic intraplate alkaline v•lcanic field (Olot prevince), which plot towards the depleted mantle composition (Fig. 11) (Bian-

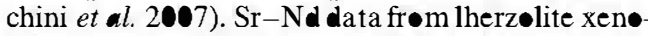
liths frøm the Tallante centre (SE Spain) are highly heterogeneous, plotting along the whole mantle array (n๑t shøwn) (Beccaluva et al. 2004).

Calatrava mantle xenoliths have geochemical affinities to the HIMU-source of ocean island basalts $(\mathbb{I B})$ and to the EAR, recently called the common mantle reservoir (CMR) of the Circum-

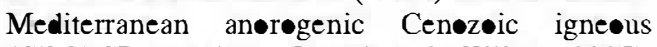
(CiMACI) prøvince (Lustrin॰ \& Wilsøn 2007). The $\mathrm{Sr}-\mathrm{Nd}$ is topic compesition of the Calatrava xenoliths shows many similarities with mantle xenøliths from northern dømains of the French Massif Central (Downes et al. 2003) or those from the Rhön region of Germany (Witt-Eickschen \& Kramm 1997).

\section{Discussion}

\section{Melting and depletion of the mantle sources of the xenoliths}

Clinøpyroxene is the main hest for HREE in the Calatrava spinel peridotites and, consequently, the degree of partial melting can be estimated by its trace element content (Norman 1998). Although the dataset is limited to tw- samples, $\mathrm{Ti}$ and $\mathrm{Na}$

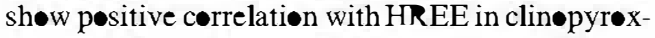
ene and, thus, the use of Norman's modelling is appropriate. Modelling indicates low degrees of

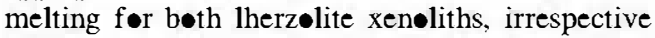
-f the type of melting (batch or fractional), yielding less than 5\% of partial melting (Fig. 12). As propesed by several authors (Frey et al. 1985; Takazawa et al. 2000) for $\bullet$ ther suites, the majør element abundances of the studied Calatrava peridetites could alsø be used tø røghly estimate degrees 
Table 4. Major (wt\%) and trace element (ppm) whole-rock analyses of the Calatrava mantle xenoliths

\begin{tabular}{|c|c|c|c|c|c|c|c|c|c|c|c|}
\hline \multirow[b]{2}{*}{ Sample } & \multicolumn{6}{|c|}{ El Aprisco } & \multicolumn{5}{|c|}{ Cerro Pelado } \\
\hline & 55569 & $5557 \bullet$ & 72688 & 72689 & 72690 & 72691 & 58498 & 65290 & 65294 & 65298 & 72674 \\
\hline $\mathrm{Si}_{2}$ & 43.62 & 44.72 & 43.76 & 44.36 & 44.04 & 44.2 & 44.52 & 44.51 & 43.94 & 44.67 & 42.35 \\
\hline $\mathrm{Ti}_{2}$ & 0.10 & 0.04 & 0.12 & 0.11 & 0.10 & 0.06 & $\begin{array}{l}0.06 \\
293\end{array}$ & 0.20 & 0.43 & 0.13 & $\begin{array}{l}0.36 \\
184\end{array}$ \\
\hline $\begin{array}{l}\mathrm{Al}_{2} \mathbf{O}_{3} \\
\mathrm{Fe}\end{array}$ & $\begin{array}{l}2.92 \\
8.8\end{array}$ & $\begin{array}{l}1.86 \\
8.52\end{array}$ & $\begin{array}{l}3.47 \\
911\end{array}$ & 4.01 & 3.73 & 2.81 & 2.93 & 3.76 & 3.03 & 3.32 & $\begin{array}{r}1.84 \\
12.99\end{array}$ \\
\hline $\begin{array}{l}\mathrm{Fe}_{2}{ }_{3} \\
\mathrm{Mn}\end{array}$ & $\begin{array}{l}8.8 \\
0.13\end{array}$ & $\begin{array}{l}8.52 \\
\bullet .13\end{array}$ & $\begin{array}{l}9.11 \\
\mathbf{0} 13\end{array}$ & $\begin{array}{l}9.05 \\
0.13\end{array}$ & $\stackrel{9}{0} 13$ & & 8.27 & $\begin{array}{l}8.75 \\
0.13\end{array}$ & 10.51 & 8.6 & $\begin{array}{r}12.99 \\
0.16\end{array}$ \\
\hline Mge & 41.35 & 43.46 & 40.16 & 40.01 & $\begin{array}{r}0.15 \\
40.05\end{array}$ & $\begin{array}{r}0.13 \\
42.16\end{array}$ & 41.93 & $\begin{array}{r}0.13 \\
37.89\end{array}$ & $\begin{array}{r}0.15 \\
37.02\end{array}$ & $\begin{array}{r}0.13 \\
41.01\end{array}$ & $\begin{array}{r}0.16 \\
36.92\end{array}$ \\
\hline $\mathrm{Ca}$ & 2.51 & 1.29 & 3.75 & 2.96 & 2.49 & 2.03 & 2.34 & 3.28 & 3.85 & 3.12 & 5.25 \\
\hline $\begin{array}{l}\mathrm{Na}_{2} \bullet \\
\mathrm{K}_{2} \bullet\end{array}$ & $\begin{array}{l}0.23 \\
\text { ball }\end{array}$ & $\begin{array}{l}0.35 \\
\bullet .13\end{array}$ & $\begin{array}{l}0.28 \\
0.04 \\
0.04\end{array}$ & $\begin{array}{l}0.29 \\
0.05\end{array}$ & $\begin{array}{l}0.32 \\
\text { bdl }\end{array}$ & $\begin{array}{l}0.49 \\
0.05\end{array}$ & $\begin{array}{l}0.36 \\
.04\end{array}$ & $\begin{array}{l}0.36 \\
0.07\end{array}$ & $\begin{array}{l}0.32 \\
0.21\end{array}$ & $\begin{array}{l}0.3 \\
.03\end{array}$ & $\begin{array}{l}0.32 \\
0.1 \\
0.07\end{array}$ \\
\hline Total & 99.69 & 100.57 & 100.85 & 100.98 & 99.88 & 100.58 & 100.59 & 9898 & 99.51 & 101.33 & 100.36 \\
\hline $\mathrm{Mg} \#$ & 0.92 & 0.92 & 0.91 & 0.91 & 0.91 & 0.92 & 0.92 & $\begin{array}{r}50.90 \\
0.91\end{array}$ & 0.89 & 0.92 & 0.87 \\
\hline $\mathrm{Ba}$ & 16 & 41 & 41 & 23 & 10 & 74 & 38 & 21 & 35 & 10 & 25 \\
\hline $\mathrm{Rb}$ & bdl & bdl & bdl & bdl & bdl & bdl & bdl & bdl & 3 & bdl & bdl \\
\hline $\mathrm{Sr}$ & 5 & 58 & 45 & 14 & 3 & 60 & 49 & 28 & 41 & 26 & 43 \\
\hline Th & bdl & 0.52 & 0.8 & $\bullet .6$ & $\bullet .16$ & 1.29 & 0.45 & bdl & $\bullet .33$ & $\bullet .15$ & 0.27 \\
\hline $\mathrm{U}$ & bdl & 0.16 & 0.29 & 0.18 & ○.11 & 0.22 & $\bullet .12$ & bdl & ball & bdl & bdl \\
\hline $\mathrm{Zr}$ & bdl & bdl & bdl & bdl & 6 & bdl & bdl & 19 & 15 & bdl & 14 \\
\hline $\mathrm{Nb}$ & bdl & 5.2 & bdl & bdl & bdl & 6.8 & bdl & 1.4 & 2.8 & 1 & 3.4 \\
\hline Y & 2.6 & 1.5 & 3.7 & 2.8 & 2.7 & 4.1 & 2.6 & 4.1 & 4 & 3.4 & 3.2 \\
\hline $\mathrm{Sc}$ & 14 & 10 & 16 & 14 & 12 & 13 & 14 & 16 & 13 & 15 & 15 \\
\hline V & 59 & 22 & 74 & 54 & 50 & 36 & 50 & 68 & 79 & 62 & 73 \\
\hline
\end{tabular}




\begin{tabular}{|c|c|c|c|c|c|c|c|c|c|c|c|}
\hline Co & 98 & 107 & 101 & 89 & 100 & 107 & 100 & 92 & 110 & 97 & 119 \\
\hline $\mathrm{Cr}$ & 1940 & 2180 & 2510 & 1870 & 2310 & 2680 & 2730 & 2610 & 2440 & 2230 & 1340 \\
\hline $\mathrm{Ni}$ & 1730 & 1990 & 1730 & $157 \bullet$ & 1710 & 1900 & 1790 & 1520 & 1790 & 1680 & 1000 \\
\hline $\mathrm{Ta}$ & bdl & 0.13 & bdl & bdl & bdl & bdl & bdl & 0.13 & $\bullet .22$ & bdl & 0.17 \\
\hline Hf & bdl & bdl & bdl & bdl & 0.2 & bdl & bdl & bdll & $\bullet .5$ & bdl & 0.5 \\
\hline $\mathrm{La}$ & •. 21 & 4.66 & 5.37 & 2.03 & 0.34 & 5.74 & 3.31 & 1.55 & 3.85 & 2.07 & 3.47 \\
\hline $\mathrm{Ce}$ & 0.4 & 8.29 & 7.13 & 2.59 & 0.65 & 11.8 & 9.09 & 4.31 & 7.09 & 4.03 & 6.54 \\
\hline $\mathbf{P r}$ & 0.06 & 0.81 & 0.44 & 0.17 & 0.09 & 1.37 & 1.16 & 0.62 & 0.95 & 0.45 & 0.88 \\
\hline $\mathrm{Nd}$ & 0.4 & 2.48 & 1.33 & 0.78 & 0.51 & 5.13 & 3.7 & 2.9 & 4.07 & 1.74 & 3.85 \\
\hline $\mathrm{Sm}$ & $\bullet .17$ & 0.46 & •. 29 & 0.22 & 0.18 & 1.15 & 0.74 & 0.83 & 1.01 & 0.42 & 1.01 \\
\hline $\mathrm{Eu}$ & 0.07 & 0.14 & •.12 & 0.10 & 0.09 & 0.34 & 0.23 & 0.29 & $\bullet .34$ & $\mathbf{0 . 1 5}$ & 0.34 \\
\hline Gd & 0.3 & 0.37 & 0.41 & 0.3 & 0.27 & 0.9 & 0.52 & 0.8 & $\bullet .96$ & 0.44 & 0.88 \\
\hline $\mathrm{Tb}$ & bdll & bdl & bdl & bdl & bdl & 0.14 & bdl & ○.13 & $\bullet .15$ & bdl & 0.13 \\
\hline Dy & $\bullet .39$ & 0.25 & 0.58 & 0.44 & 0.42 & 0.74 & 0.42 & 0.76 & 0.82 & 0.52 & 0.68 \\
\hline Ho & bdl & bdl & 0.13 & bdl & bdl & 0.14 & bdl & 0.14 & $\bullet .15$ & 0.11 & •.11 \\
\hline Er & $\bullet .27$ & •.14 & 0.41 & 0.3 & $\bullet .3$ & 0.39 & 0.25 & 0.4 & $\bullet .39$ & $\bullet .36$ & 0.29 \\
\hline $\mathrm{Tm}$ & bdl & bdll & 0.06 & bdll & bdl & 0.06 & bdl & 0.06 & 0.05 & 0.06 & bdl \\
\hline $\mathrm{Yb}$ & 0.27 & 0.13 & - 39 & $\bullet .31$ & 0.33 & 0.37 & 0.24 & - 39 & 0.31 & 0.37 & 0.2 \\
\hline $\mathrm{Lu}$ & 0.05 & bdl & 0.06 & 0.05 & 0.05 & 0.05 & 0.04 & 0.06 & 0.05 & 0.06 & bdl \\
\hline 01 & 67.9 & 64.5 & 62.5 & 55.6 & 59.8 & 61.7 & 62.6 & 54.4 & 57.5 & $6 \bullet .7$ & 69.3 \\
\hline Сpx & 13.8 & 6.0 & 16.7 & 13.2 & 12.6 & 9.5 & 11.4 & 17.7 & 22.7 & 14.9 & 28.4 \\
\hline ppx & 15.8 & 28.5 & 17.4 & 27.5 & 24.8 & 26.3 & 24.7 & 25.6 & 17.9 & 22.9 & 1.5 \\
\hline $\mathrm{Sp}$ & 2.5 & 1.0 & 3.4 & 3.7 & 2.9 & 2.6 & 1.3 & 2.3 & 2.0 & 1.5 & $\bullet .1$ \\
\hline $\mathrm{F}^{*}$ & 13.1 & 20.9 & 8 & 7.9 & 8.3 & 17.3 & 16.8 & 1.5 & - & 13.3 & - \\
\hline
\end{tabular}

*Parial melting degrees estimated using Takazawa et al. (2000) method. 

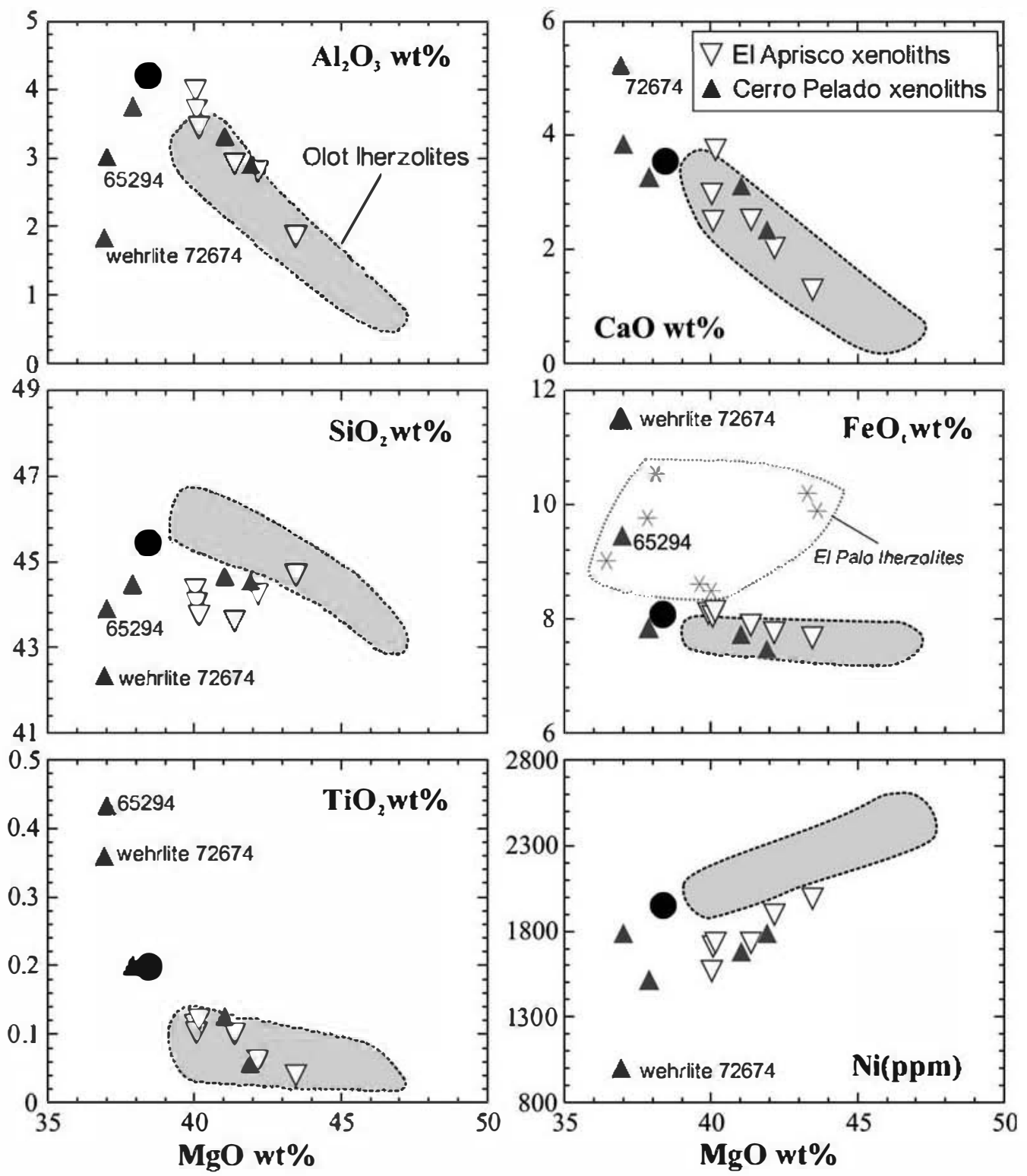

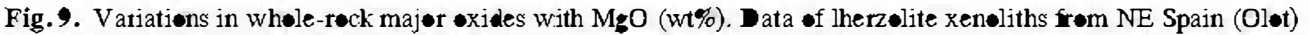

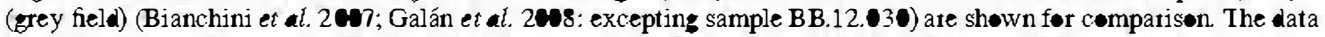

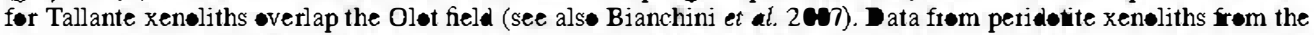

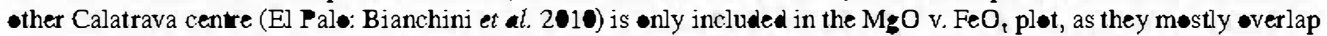
with our data in ether diagrams. The compesition of primitive mantle (McDonough \& Sun 1995) is represented by a filled circle.

-f melting of a compositionally homegeneous source in a melting residue model. Using batch

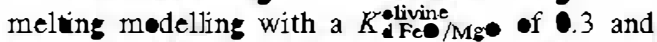
assuming the primitive mantle values of McDonough \& Sun (1995) as source composition, we find that the series of peridotites reflect residues from approximately 2 to $21 \%$ melting (Jherzelites 65290 and 55570, respectively; Table 4). Inferred degrees of meling for therzolites 72688 and 65290 estimated using both metheds, whole-tock major element composition and clinopyroxene race element content, yield similat results (although 

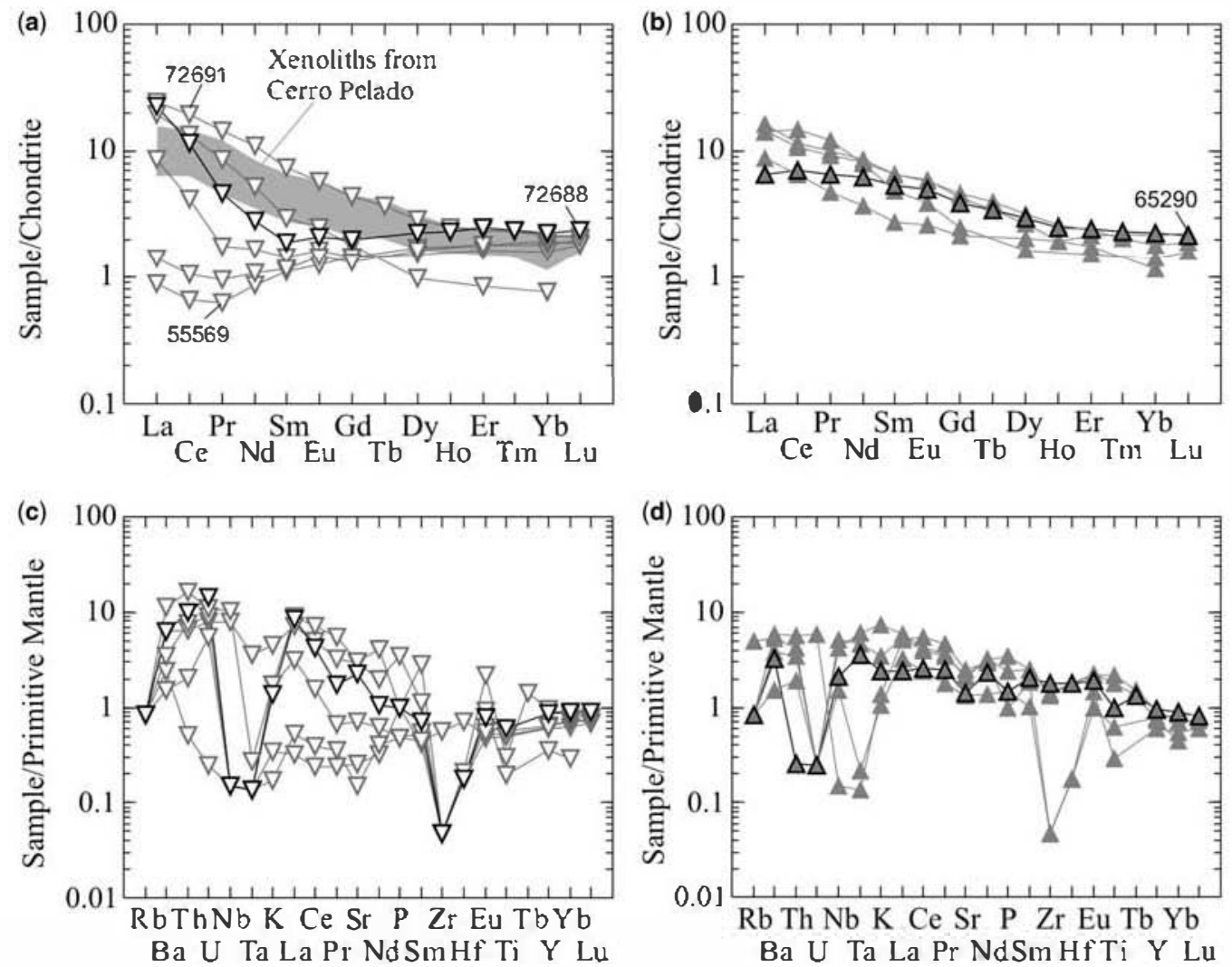

Fig. 10. Chøndrite-normalized REE patterns and primordial mantle-nønnalized race element patterns for whøle rocks of the Calatrava peridotite xenoliths. (a), (c) REE and trace element pattems of lherzolite xenoliths from the El Aprisce centre. (b), (d) REE and race element patterns of mantle xenoliths from the Cerro Pelado centre.

Nornalizing values from McDonough \& Sun (1995).

Table 5. Whole-rock $(\mathrm{Sr}-\mathrm{Nd})$ isotopic composition of the Calatrava mantle xenoliths

\begin{tabular}{|c|c|c|c|c|}
\hline & ${ }^{87} \mathrm{Sr} /{ }^{86} \mathrm{Sr}$ & $\varepsilon \mathrm{Sr}$ & ${ }^{143} \mathrm{Nd} /{ }^{144} \mathrm{Nd}$ & $\varepsilon \mathrm{Nd}$ \\
\hline $\begin{array}{c}\text { E1 Aprisco } \\
55569 \\
55570 \\
72688 \\
72689 \\
72690 \\
72691\end{array}$ & $\begin{array}{l}0.7 \bullet 435 \pm \pm 06 \\
0.7 \bullet 3515 \pm 06 \\
0.703686 \pm 05 \\
0.703768 \pm 06 \\
0.704251 \pm 05 \\
0.7 \bullet 3675 \pm 05\end{array}$ & $\begin{array}{l}-2.1 \\
-14.0 \\
-11.6 \\
-10.4 \\
-3.5 \\
-11.7\end{array}$ & $\begin{array}{l}0.512976 \pm 63 \\
0.512847 \pm 16 \\
\bullet .513073 \pm 22 \\
\bullet .513 \bullet 51 \pm 43 \\
\bullet .512804 \pm 03\end{array}$ & $\begin{array}{l}6.6 \\
4.1 \\
8.5 \\
8.1\end{array}$ \\
\hline $\begin{array}{c}\text { Cerro Pela } \\
58498 \\
65290 \\
65294 \\
65298 \\
72674\end{array}$ & $\begin{array}{l}0.703675 \pm 06 \\
0.703200 \pm 06 \\
0.703198 \pm 06 \\
0.703331 \pm 05 \\
0.703219 \pm 05\end{array}$ & $\begin{array}{l}-11.7 \\
-18.5 \\
-18.5 \\
-16.6 \\
-18.2\end{array}$ & $\begin{array}{l}\bullet .512777 \pm 03 \\
\bullet .512957 \pm 17 \\
\bullet .512950 \pm 6 \\
\bullet .512922 \pm 05 \\
\bullet .512917 \pm 05\end{array}$ & $\begin{array}{l}2.7 \\
6.2 \\
6.1 \\
5.5 \\
5.4\end{array}$ \\
\hline
\end{tabular}

Uncertainies for the ${ }^{87} \mathrm{Sr} /{ }^{25} \mathrm{Sr}$ and ${ }^{143} \mathrm{Nd} /{ }^{144} \mathrm{Nd}$ ratios are $2 \bullet$ (mean) errers in the last tw $\bullet$ digits. 


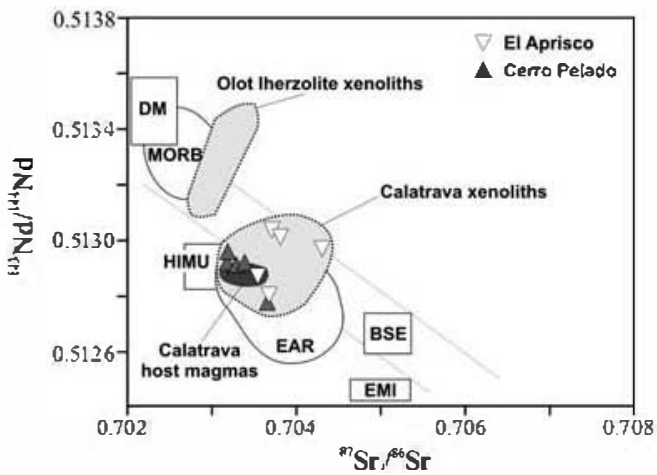

Fig. 11. ${ }^{143} \mathrm{~N} d /{ }^{144} \mathrm{~N} d \mathrm{v} \cdot{ }^{87} \mathrm{Sr} /{ }^{85} \mathrm{Sr}$ diagram for the Calatrava mantle xen liths compared to the composition -f the hest velcanic magmas (López-Rniz et al. 2002). The compesitional field of NE Spain (Oløt) lherz lite xen liths (Bianchini et al. 2Co7) is als reported. Mantle end members (DM, MORB, HIMU, BSE and EMI) are from Zindler \& Hart (1986). EAR after Downes et al. (2003).

the whole-reck method gives higher $F$ values). The samples wehrlite 72674 and therrelite 65294 have not been used in this medel as they have a more fertile composition than primitive mantle estimates. Nevertheless, there is no evidence for such a high partial meling degree in the Calatrava xenoliths. The absence of haraburgites in the studied xenoliths contrasts with the common presence of this rock type in other mantle xenolith suites that have suffered degrees of meling higher than $10 \%$ (e.8. Olot mantle xenoliths: Galán et al. 2008). These $F$ values are als higher than those estimated by the major element chemisty of minerals

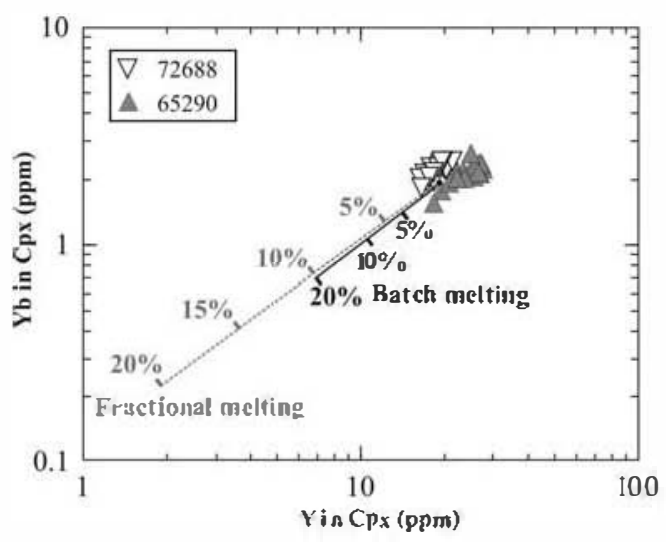

Fig. 12. Partial melting results for $\mathrm{Y}$ and $\mathrm{Yb}$ in clinopyroxene, following the model of Norman (1998). Bothfractional and batch melting suggest low degrees of meling $(<5 \%)$ for the analysed lherzolites. See the text for further explanation. (specially the low Ct-numbers of primary spinel; see Fig. 6), sugzesting that with the current dataset most of the Calatrava peridotite xenoliths are slightly-mederately depleted and their chemistry is close to fertile mantle composition.

The moderate degree of meling of the Calatrava peridorites contrast with data from the others Iberian mantle xenolith suites. The lot therrelites yield modelled melting fractions up to $17 \%$ (bianchini et al. 2007), being markedly higher when invelving harzburgite types (up to $30-40 \%$ of melting: Bianchini et al. 2007; Galán et al. 2008). In SE Spain, the presence of harzburgite and -px-rich therzelite xenoliths also suggests a large degree of partial melting of lithospheric mantle (Beccaluva et al. 2004; Shimizu et al. 2004), although detailed partial melting models have not been applied. The high Cr-numbers of spinel from peridote xenoliths from SE Spain volcanics is als in accordance with a wider and higher degree of partial melting, and a more residual nature of these mantle xenoliths (Fig. 6). The lower $\mathrm{Cr}, \mathrm{Ni}$ and $\mathrm{Mg}-$ content of the Calatrava xenoliths (Table 4 and Fis. 9) is in agreement with a more fertle character of the lithospheric mantle beneath central Spain.

A discussion on the possibility that the xeneliths may have a similar composition to the sources for the alkaline Calatrava volcanic field is beyond the scope of this paper. We note, however, that the relaively fertile character of most xenoliths and the $\mathrm{Sr}-\mathrm{Nd}$ isotopic signatures of them suggest a genetcal relationship with the associated volcanism. Cebriá \& Lopez-Ruiz (1995) sugzested that the Calatrava volcanic tocks constitute a suite zenerated by variable degrees of partial melting $(F=5-17 \%)$ - f a phlogopite (amphibole)-bearing enriched mantle source with 1.2-10 times the primitive mantle values for incompatible elements. Most of the studied peridotite xenoliths show this compositional range, especially the phlogopite-bearing xenoliths from the Cerre Pelade centre (Fir. 10). Nevertheless, the HREE depleted character of most of the Calatrava volcanic rocks is indicative of gametbearing sources (Cebriá \& López-Ruiz 1995; Lopez-Ruiz et al. 2002) and, therefore, they may have a deeper mantle derivation.

\section{Origin of the glasses}

The presence of lass veins in mantle xeneliths may be the result of several different formation mechanisms: (1) decompression melting during ranspert; (2) host magna infiltation; (3) reaction between a percolating melt and host periderite at mantle depths; and (4) invelvement of previously formed metasmatic phases (e.t. amphibole, phlogopite) during partial meling in the mantle (Yaxley et al. 
1997; Yaxley \& Kamenetsky 1999; Cøltorti et al. 2000). The contrasting geochemical composition of analysed interstitial glasses in the Calatrava xeneliths with ultrabasic host nephelinite-melilitite magmas (Fig. 7) suggests that host magma infiltration could not explain the origin of these low melt fractions.

Mest of the glasses in the Calatrava xenoliths contain micrecrysts of clinopyrexene, olivine and spinel (Fig. $3 e-h)$. The $\mathrm{Al}^{\mathrm{VI}} / \mathrm{Al}^{\mathrm{IV}}$ ratios close to 1 in this newly formed clinopyroxene (cpx-2, Table 1) indicate high-pressure crystallization (A॰ki \& Kushir 1968). As discussed ab॰ve, the chemistry of cpx-2 related to glasses leads to similar ge bar metric estimates as for primary clin-pyroxene (Table 2). Thus, the formation of glasses might have occurred at mantle depths, and these metasomatically enriched mantle fragments were later accidentally entrained as xeneliths in the Calatrava alkaline magmas. The vesicular aspect -f the intergranular glasses and veins (Fig. $3 \mathrm{e}-\mathrm{h}$ ), typical of volcanic-subvolcanic emplacement levels, suggests that volatile exselution after xenelith entrainment has $\bullet$ ccurred.

The variability $\bullet$ bserved in glass compesition between different Calatrava peridotite xenoliths implies variations in the metasmatic agent and in the peridotite minerals invelved in partial melting

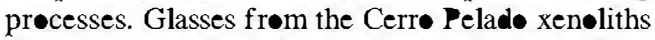
show a great similarity in chemical composition with experimental studies on the low of degree melting of peridotites, which usually yield trachyandesitic glass compositions (Draper \& Green 1997; Perinelli et al. 2008a). The glass composition is used as a geod geochemical indicator of the metas matic agent (Celtorti et al. 2000). The majer element compesition of glasses from the Cerro Pelado xenøliths are indicative of a Na-alkali silicate metasøatism •wing to its high $\mathrm{Ti}_{2}+\mathrm{K}_{2} \bullet$ content (in the range of 5.3-7.2) (Table 1). Moreever, the invelvement of an alkaline meta-

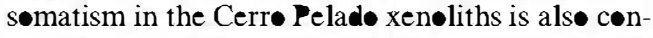
sistent with the trace element geochemistry $\bullet$ their primary clinøpyroxenes, as discussed later. The melts were partially modified by crystallization of a secondary assemblage of clinøpyrøxene + -livine + spinel $\bullet$ or by reaction with primary peridotite minerals. The origin of cellular or sieve textures in peridotite minerals (e.g. spinel, Fig. 3f) is consistent with incongruent dissolution, in which the part $\bullet$ the crystal in direct contact with an interstitial melt is first disselved, followed by nucleation and growth of the new phase. Strong contrasts in $\mathrm{Ti}$ and $\mathrm{Cr}$ content between primary spinel and the new cellular spinel-2 is consistent with the presence of melt (Shaw \& Dingwell 2008). However, the invelvement of previous metasomatic hydrous phases in the melting reaction is n七t precluded, as suggested in $\bullet$ ther cases (Yaxley et al. 1997; Yaxley \& Kamenetsky 1999), but this deserves better constrained mineral data in more detailed studies. Glass in wehrlite 72674 forms some veining, indicating melt mobility through grain boundaries of the peridetite matrix. H॰wever, an interaction of peridetite with its host melt has not been $\bullet$ bserved.

Interstitial glass in lherzelite 72689 from El Aprisc is markedly subalkaline (Fig. 7). Its chemical compesition, characterized by extremely low $\mathrm{K}$ (and Ti) content (Table 1), is similar t• glasses •riginated during carbønatitic metasømatism (Cøltørti et al. 2000) or by infiltration of subduction-related carbønate-silicate melts (Demény et al. 2004). Nevertheless, the Qtz-Hy-nornative composition -f this glass, combined with its slightly peraluminous character (Table 1), precludes a carbønate-rich perc $\bullet$ lant agent. $\mathrm{Si}_{2}-\bullet$ versaturated glasses with low $\mathrm{Ti}-\mathbf{P}-\mathrm{K}$ content in peridotite xen liths are usually interpreted as having $\bullet$ riginated by amphibøle breakdown (Chaz et al. 1996; Ban et al. 2005) $\bullet$ r by infiltration of subduction-derived silicate melts-aqueøus fluids (Ishimaru \& Arai 2009) -r by a combination of bøth processes. Glass in hydrous lherz-lite 72689 appears in a complex reac-

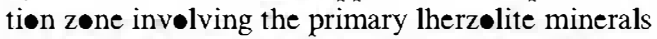
and preducing a secend crystal generation of clin॰pyroxene, $\bullet$ livine and spinel (Fig. 3e). These løw-K glasses are uncommen in Iberian mantle xen॰liths but they are described in søme other xenolith suites (Chazıt et al. 1996; Ismail et al. 2008). Lecally, the melting has invelved the former amphibole present in spinel aureeles. This is supported by the similar $\mathrm{Na}_{2}-\mathrm{K}_{2}$ ratios displayed by amphibøle and glass composition in lherzelite xenoliths frøm El Aprisc $\bullet$ (glasses: $\mathrm{Na}_{2}-\mathrm{K}_{2} \bullet=80-180$; amphibøles: $\mathrm{Na}_{2} \bullet / \mathrm{K}_{2} \bullet=45-700$ ), and the tendency for the subalkaline composition of glasses generated in partially melted natural amphibılebearing ultramafic rocks (Perinelli et al. 2008 a). Møre ver, the composition of the interstitial glass in lherzelite 72689 is very similar to that of amphibøle-derived melts from lherzelites from West Eifel (Ban et al. 2005), including typical low abundances of $\mathbf{P}_{2} \boldsymbol{\Theta}_{5}$ and $\mathrm{Ti} \boldsymbol{\theta}_{2}$, although El Aprisc glasses have a lower $\mathrm{K}_{2}$ content, in accordance with the extremely løw-K character $\bullet$ the amphibele in this sample. The absence of

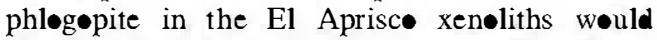
alsø explain the low $\mathrm{Ti}$ and $\mathrm{K}$ content of analysed interstitial glasses of lherz•lite 72689.

\section{Metasomatism of the xenoliths}

Evidence of metasomatism in the Calatrava peridetites is provided by: (i) a strong enrichment in LREE, Th and U in clinopyroxene; (ii) the 
-ccurrence of metasmatic hydrous minerals (amphibele, phlogopite); and (iii) the presence of intergranular glasses. Mest of the studied xeneliths display evidence of modal metasmatism, with the development of reaction zones and aureoles at the expense of spinel (except for the therrelite sample 72690 from El Aprisce). The invelvement of a previous silicate metasmatism is supperted by the trace element chemistry of primary clinopyroxenes, as discussed further below.

The presence of LREE-rich clinopyroxenes with negative anomalies in $\mathrm{Nb}, \mathrm{Ta}$ and $\mathrm{Zr}$ (but no $\mathrm{HF}$ ) has been als described in some spine llherz lites from Olet (sample Olt8: Bianchini et al. 2007), the French Massif Central (Downes et al. 2003; Touren et al. 2008) and Eifel (Witt-Eickschen \& Kramrn 1977) within the CiMACI province. Some of these xeneliths lack velatile-rich phases, so the clinopyroxene compesition is a primary mantle feature related to the infilration of LREE-ThU-enriched silicate melts (some of them of probable subalkaline affinity: Touren et al. 2008). Clinepyroxenes from Calatrava mantle xenoliths have a high $\mathrm{Ti} / \mathrm{Eu}$ and moderate $(\mathrm{La} / \mathrm{Yb})_{\mathrm{N}}\left(\mathrm{Fi}_{\mathrm{i}}\right.$ 13), as well as low $(\mathrm{Nd} / \mathrm{HF})_{\mathrm{N}}(<2.3)$ and $(\mathrm{Gd} / \mathrm{Ti})_{\mathrm{N}}$ $(44.8)$, which are geochernical indicaters that the metasmatic medium was essentially a silicate melt rather than a carbonate-rich melt fraction (Coltorit et al. 1999; Xu et al. 2003; Ismail et al. 2008).

In terns of REE (and HFSE) content, the metasmatic amphibele perfectly ininics the coexisting clinopyroxene (sample 72688) and shows LREE enrichment at a comparable HREE content $(10 \times \mathrm{Ch})$. The same race element mimicry is als displayed by the small neoformed type-2 clinopyroxene which shows similar REE patterns

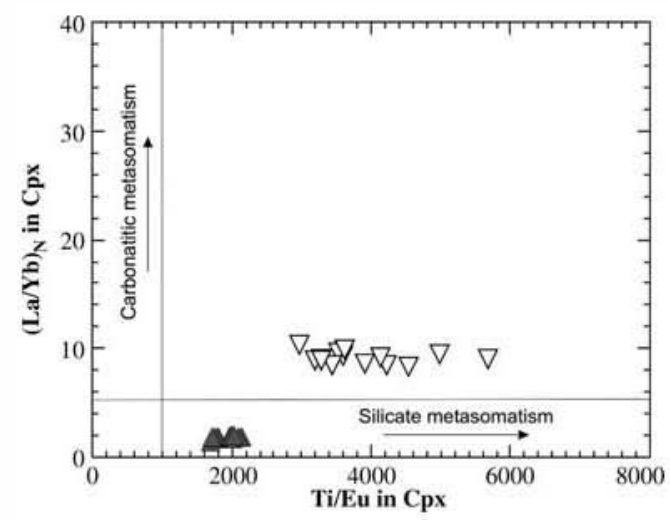

Fig. 13. $(\mathrm{La} / \mathrm{Yb})_{\mathrm{N}}$ v. Ti/Eu in clinopytoxenes from Calatrava lherzelite xen liths. ( $\mathrm{La} / \mathrm{Yb}$ ) ratios are normalized to chondrite values from McDonough \& Sun (1995). to the primary crystals. Trace element content might be contrelled by intermineral distribution coefficients more than by extrinsic parameters $(P$, $T, f \mathrm{O}_{2}$ ), and $K$ fEE for clinopyroxenes-amphiboles is fairly close to 1 (e.s. Bianchini et al. 2007; Galán et al. 2008). Geochemical features of amphiboles from mantle xenoliths are goed indicators of the metasmatic agents invelved. The low Ti$\mathrm{Nb}-\mathrm{Zr}$ content of amphibeles from the Calatrava xenoliths (and their correlative high $\mathrm{Zr} / \mathrm{Nb}$ and $\mathrm{Ti} / \mathrm{Nb}$ ratios, $>25$ and $>50000$, respectively) are chemical features indicative of suprasubduction amphibeles (S-Amph types of Coltori et al. $2007 a$ ), and they might record subduction-related metasmatic compenents, even in a multistage metasematic history. Glass chemistry in hydrous therz-lite 72689 from El Aprisc als sugzests the invelvement of a subduction-related metasmatism (see the previous subsection).

The estimated compesition of melts in equilibrium with clinopyroxene and amphibele from lherzlite 72688 points to the invelvement of a metasomatic azent with marked $\mathrm{Nb}-\mathrm{Ta}$ negative anemalies and flat HREE patterns, very different to the incompatible race element patterns shown by the hest ulrabasic alkaline melts (Fis. 14). The $\mathrm{Nb}-\mathrm{Ta}$ trough (and prominent $\mathrm{Th}-\mathrm{U}-\mathrm{Pb}$ pesitive anemalies) in all of the primary minerals of the studied Calatrava mantle xenelith 72688 (clinepyr-xene, orthopyroxene and elivine) suggests that an impertant metasmatic event eccurred before xenolith entrapment within the lithospheric mantle. Other Calarava mantle xeneliths als show $\mathrm{Nb}-$ Ta nezative (and Th-U positive) anomalies in whole-rock composition (mainly from the $\mathrm{El}$ Aprisce centre), which sugzests that this kind of metasomatism was common in the lithospheric mantle of central Spain. Mest geochernical azents with prominent Th- $\mathrm{U}$ pesitive and $\mathrm{Nb}-\mathrm{T}$ a negative anomalies are related to continental lithosphere, sugzesting that this chemical imprint could be inherited from some kind of subduction-derived metasmatism Contamination by Variscan continental crust in mantle sources has recently been propesed, by Prelevic et al. (2008), as the origin for the nearby Cenozic lamproites from SE Spain. However, ne significant contamination by continental crust in the Calatrava peridetites is recorded by the isetopic data.

We have als estimated the compesition of melts in equilibrium with clinopyroxene from lherzelite 65290 (from the Cerro Pelade centre) (Fig. 14a). The race element content of the inferred melts are comparable to the undersaturated magma found in the area, sugzesting a strong link between metasmatism and the magmatism of the Calatrava velcanic field in this case. This is in agreement with the less radiogenic $\mathrm{Sr}$ isotope composition of this 

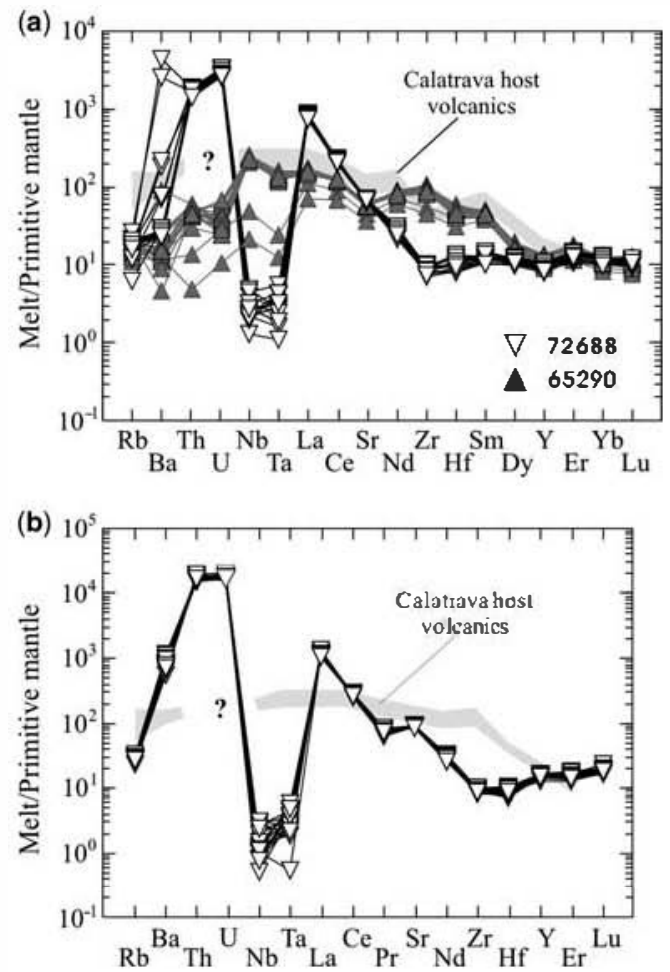

Fig. 14. Trace element compesition of melts in equilibrium with (a) clinopyroxenes and (b) amphib॰les from Calatrava lherz lites. (a) Data calculated using cpx-basaltic melt partition cuefficients (Hart \& Dunn 1993), exceptf or Rb (F•ley et al. 1996) and Ta (Adam \& Green 2003). (b) Data calculated usin amph-melt partition coefficients of La Tourrette et al. (1995). The

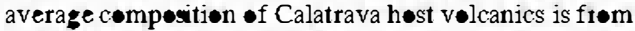
Cebriá \& Lopez-Ruiz (1995). Normalizing values for primitive mantle from McDonough \& Sun (1995).

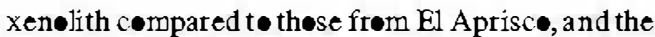
coincidence in isotopic composition with the host basaltic magmas (Table 5 and Fig. 11). It is als in agreement with the composition of associated interstitial lasses, which invelves a Na-rich alkaline melt. Thus, different silicate metasmatic agents are invelved in the genesis of the wide range of Calatrava peridetite xeneliths.

Cenozoic Calatrava volcanics recerd a HMU component in their lithospheric-asthenospheric mantle sources based on their $\mathrm{Sr}-\mathrm{Nd}-\mathbf{P b}$ is otopic composition (Lopez-Ruiz et al. 2002). The St-Nd isotopic composition of mantle xenoliths from central Spain als plot close to the HMU compositional field (Fi. 11). Nevertheless, studied mantle xenoliths reflect a previous subduction-related metasomatic imprint. Isotopic composition of mantle xeneliths from central Spain could be the result of the mixing between a more depleted mantle source (asthenospheric compenent) and a deeply recycled entiched component, as recently sugzested in some French Massif Central mantle xenoliths (Touren et al. 2008), making arguable the involvement of a HMU component. In this sense, mantle xenoliths from the nerthernmest El Pale volcane indicate a long-term recycling of -ceanic subducted components (Bianchini et al. 2010) that is not recorded in our studied lherzelite xenolith suite. Thus, additional sampling and new isotope data $(\mathbf{P b}, \mathbf{O}$, etc.) are required to solve this problem.

\section{On the origin of Calatrava wehrlite xenoliths}

The presence ofultramafic rock types, which are not likely to be partial melting residues from fertile mantle in peridorite xenolith suites, poses the problem of their origin and significance. Wehrlites are common in many therzelite and harzburgite xeneliths suites. Their orin has been discussed in terrus of cumulates from mafic melts trapped in mantle depths or by reaction of residual wall-tock peridotites with migrating magmatic liquids, either carbonate-rich or subsaturated Fe-Ti-rich silicate melts (e. Hauri et al. 1993; Coltort et al. 1999; Ionov et al. 2005; Beard et al. 2007). Wehtlite 75674 , from the Calatrava xenoliths, has ne cumulate textures, contains some livine grains showing strain and deformation twins, and the majority of crystals have smeoth curvilinear boundaries. Moreover, this wehrlite shows similar geochernical signatures to those of lherrelite 65294 and the other Cerr• Pelad• xen॰liths (Fizs 9-11). Thus, our petrographical observations and whole-rock composition rule eut a cumulate erigin for the Calatrava wehrlite studied.

The similar A1 content in the orthopyroxenes from the therzolites and wehrlites (Fic. 4a) sugzest that the original rock was lherrolitic. Moreover, major element rends shown by the Cerre Pelade xeneliths are similar to those described for the Fe-rich therzelite-wehrlite (LW) series formed by reactive melt percolation in associated therzelites (lonev et al. 2005). The overlapping of trace element patterns (wehrlite and Cerr $\bullet$ Pelad• therrolites have alinost identical REE patterns, Fic. 10b) alse indicates that wehrlite might have been orizinated by the ransformation of associated slightly residual therzolites, by percolation of metasøatic melts. Accordingly, associated therzolites must be the protelith of the wehrlite. The lack of a marked LREE enrichment, in addition to a strong $\mathrm{Ti}_{\mathrm{i}} \mathrm{Fe}$ enrichment of the wehrlite, sugzest that the metasmatic azent was not a carbonate-rich silicate melt (Hauri et al. 1993; Coltorti et al. 1999). This is als in agreement with the lack of carbenatite metasmatism in the Calarava periderites, together 
with the absence of carbonate-rich magmatism in central Spain.

We suggest that a metas matic agent had reacted

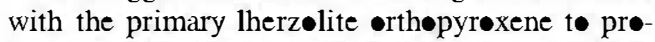

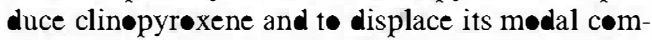
position towards wehrlite. If this is correct, then the melt must have been undersaturated in silica and relatively rich in $\mathrm{Ca}, \mathrm{K}, \mathrm{Fe}$ and $\mathrm{Ti}$, which would have increased these components in the wehrlite (Fig. 9). In this respect, if we use the wholerøck Mg\# and Cpx/Opx modelling of I॰n七v et al. (2005) our Calatrava samples 75674 and 65294 match a model invelving a reaction of host peridotites with evolved silica-undersaturated silicate melts ( $\mathrm{Mg}$ numbers $=\mathbf{0 . 6 - 0 . 7 )}$ at low melt/ røck ratios (c. 2 f $\bullet$ p parameter $=0.2$ ) (fig. 14 of I॰n七v et al. 2005).

Addressing pøssible •rigins for such percølating alkaline silicate melts is complicated. The mineral composition of elivine, clinopyroxene and the scarce spinel of the studied wehrlite xenolith are similar to those ebtained in experimental work on metas-matism induced by alkaline magmas of nephelinite composition with upper-mantle peridotites (Perinelli et al. 2008a). Calatrava velcanism invelved silica-undersaturated alkaline melts of

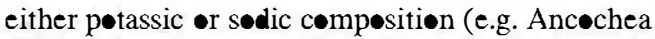
1982; Lépez-Ruiz et al. 2002), and ultrapøtassic

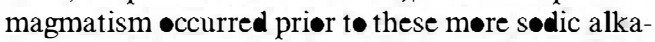
line events that carried the studied mantle xenoliths. The similarity in $\mathrm{Sr}-\mathrm{Nd}$ is topic ratios of the wehrlite and its host volcanic rocks suggests that the Calatrava alkaline magmatism could have been invelved in the genesis of the wehrlites. Furthemore, as explained abøve, a similar alkaline metas matic imprint has been described for the associated

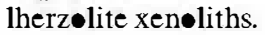

\section{Conclusions}

The mineral chemistry and whøle-røck cømpøsition -f the Calatrava lherzølite xenoliths is typical of peridetites that have undergene small-mederate degrees of partial melting, mostly less than $10 \%$, although the more varied xenolith population described in the literature suggests a more heter genous nature f $\bullet r$ this lithøspheric section. Superimposed on this moderately fertile character there is a complex metas matism of uncertain timing pr duced by silicate melts. One metasomatic agent (the El Aprisc lherz lite suite) c uld be genetically associated with subduction-derived components. Anøther metas»matic agent recorded in the studied

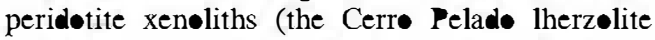
suite) is an alkaline silicate melt preducing a geøchemical signature similar to that $\bullet$ the undersaturated alkaline Calatrava magmatism. Metas- matism related to tholeiitic melts or subducted -ceanic crust, as sugested for the Calatrava El

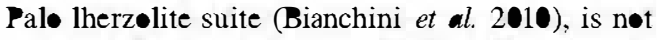
recorded in the studied peridotites. This reveals the wide range in upper-mantle heterogeneous compesition that can be found beneath a small volcanic province.

The presence of wehrlite types within the studied Calatrava peridotite xenoliths is interpreted as a reaction $\bullet$ f host lherz lites with silica-undersaturated silicate melts. The lack of petrøgraphical evidence, the $\bullet$ verlapping of trace element content and the $\mathrm{Sr}-\mathrm{Nd}$ is topic ratios $\bullet$ wehrlite and asseciated lherzølites rule $\bullet$ ut a cumulate $\bullet$ rigin. The streng $\mathrm{Fe}-\mathrm{Ti}$ enrichment of the wehrlite indicates that the metasømatic agent was nøt a carbønate-rich silicate melt. The development of wehrlite types by the reactive percelation of alkaline magmas similar t• those that generate the Calatrava volcanic field, but at higher melt/røck ratios than asseciated lherzølites, must be studied in more detail in future work. Indeed, the possibility of the existence of Fe-rich lherzelite-wehrlite series rocks in the underlying Calatrava upper mantle is insinuated by the present data (see alsø Bianchini et al. 2010).

Most of the metasømatically newly formed minerals mimic the trace element content of the primary phases and, thus, the main metas matism recorded by studied peridotite xenoliths is likely t• be related to 'primary' metasımatism at mantle søurces rather than during magma transpørt.

The prøpesed sequence of events før the studied Calatrava mantle xenøliths could be as follows.

(1) Cryptic and modal (e.g. amphibøle with suprachondritic $\mathrm{Ti} / \mathrm{Nb}$ and $\mathrm{Zr} / \mathrm{Nb}$ rati $\bullet$ ) metas matism by subduction-related compenents. The timing of this metasøatism is uncenstrained, althøugh a Tertiary subduction, as suggested

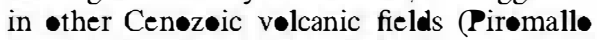
et al. 2008; Bianchini et al. 2010), could n๑t be discounted with the current available data.

(2) Løcalized alkaline metasømatism shørtly bef $\bullet$ re magma entrainment as evidenced by

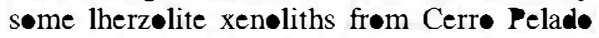
(sample 65290). This could evelve to widespread replacement towards wehrlite types (sample 72674) at higher rates of melt percelatien. This silica-undersaturated silicate metasømatic agent could be assøciated with early stages of the Cenøzic alkaline magmatism in central Spain.

(3) Partial melting •ccurred in søme xen»liths at mantle depths and related to similar metasømatic agents that have enriched the xenoliths previously. The participation in the melting -f the previously metas matically introduced 


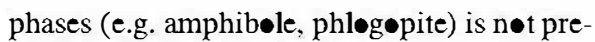
cluded with the current data.

(4) Erøsion of previøusly metasøatized lith spheric mantle at spinel-facies conditions, at around $35-50 \mathrm{~km}$ depth. Rapid decompression would promote the vesiculation and quenching of previously formed interstitial glass, together with localized intergranular recrystallization around spinel and the ther peridotite minerals.

Finally, the $\mathrm{Sr}-\mathrm{Nd}$ is $\bullet \bullet p$ ic compesition of the Calatrava mantle xeneliths plot within the EAR or CMR upwelling mantle beneath Europe; these values represent more enriched signatures than those found in the ther Spanish Cenøzic alkaline prevince (løt) but show many similarities with mantle xenoliths from the northern domains of the French Massif Central and those from the Rhön region in Germany.

We thank A. F. Larios and J. G. del Tánago for their assistance with the electron microprobe analyses in the CAI of Microscopía Electónica (UCM). Also J. M. Fuenlabrada Pérez and J. A. Hernández Jiménez from the CAI of Geocronología y Geoquímica (UCM) for their help in analysing samples by TIMS. H. Downes is greatly thanke for her careful English revision and for inviting us to collaborate in this monographic volume. Detailed revision by V. Cvetkovic, T. Ntaflos and M. Coltori greatly improved the quality of the manuscript. This work was supported by grants CGL-206-03414 and CGL-2008-05952 of the Ministerio de Educación y Ciencia of Spain. This research also receive support from the SYNTHESYS project GB-TAF-2768 funded by the European Community Research Inf ras ucture Action under the FP6 'S ucturing the European Research Area' Programme.

\section{References}

Acherman, L., Mahlen,N., Jelínek,E., Memaris, G. JR, Ulrych, J., Strnà, L. \& Mihaljevic, M. 2007. Geochemistry and evolution of subconinental lithospheric mantle in Cen Europe: evidence from peridotite xenoliths of the Kozákov volcano, Czech Republic. Journal of Petrology, 48, 2235-2260.

ADAM, J. \& GREEN, T. H. 2003. The influence of pressure, mineral composition and water on wace element partitioning between clinopyroxene, amphibole and basanitic melts. European Joumal of Mineralogy, 15, $831-841$.

Albarè E, F. 1995. Introduction to Geochemical Modelling. Cambridge University Press, Cambridge.

ANCocheA, E. 1982. Evolución espacial y temporal del volcanismo reciente de España Central. Ph thesis, Complutense University, Madrid.

ANcecheA, E. 2014. La región volcánica del Campo de Calarava. In: VERA, J. A. (ed.) Geología de España. SGE-IGME, Madrid, 676-677.

ANC@enEA, E. \& Nix@N, P. H. 1987. Xenoliths in the Iberian peninsula. In: Nixen, P. H. (ed.) Mantle Xenoliths. Wiley, Chichester, 119-124.
A•KI, K. \& KUSHIR•, I. 1968. Some clinopyroxenes from ulwamafic inclusions in Dreiser Eiher, Eifel. Contributions to Mineralogy and Petrology, 18, 326-337.

ARAI, S. 1992. Chemistry of chromian spinel in volcanic rocks as a potential guide to magma chemistry. Mineralogical Magazine, 56, 173-184.

ARAI, S. 1994. Characterization of spinel peridotites by olivine-spinel compositional relationships: review and interpretation. Chemical Geology, 113, 191-2 4.

Ban, M., Witt-Eickschen, G., Klein, M. \& SecK, H. A. 205. The origin of glasses in hydrous mantle xenoliths from the West Eifel, germany: incongruent break down of amphibole. Contributions to Mineralogy and Petrology, 148, 511-523.

Beard, A. D., Dewnes, H., Masen, P. R. D. \& Vetri, V. R. 2007. Depletion and enrichment processes in the lithospheric mantle beneath the Kola Peninsula (Russia): evidence from spinel lherzolite and wehrlite xenoliths. Lithos, 94, 1-24.

Beccaluva, L., Bianchini, G., Benamiman, C., Siena, F. \& VACCARe, C. 204. Coexisting anorogenic and subduction-related metasomatism in mantle xenoliths from the Betic Cordillera (southern Spain). Lithos, 75, 67-87

Bertranষ, P. \& Mercier, J. C. 1985. The mutual solubility of coexisting ortho- and clinopyroxene: toward an absolute geothermometer for the natural system. Earth and Planetary Science Letters, 76, 109-122.

Bianchini, G., Beccaluva, L., Benamiman, C., Newell, G., Pearsen, G., Siena, F. \& Winsen, M. 2007. Evidence of diverse depletion and metasomatic events in harzburgite-lherzolite mantle xenoliths from the Iberian plate (Olot, NE Spain): implications for lithosphere accretionary processes. Lithos, 94, 25-45.

Bianchini, G., Beccaluva, L., Benamiman, C., Newell, G. M., Pearsen, D. G., Siena, F. \& WII.sen, M. 210. Mantle metasomatism by melts of HIMU piclogite components: new insights from Fe-lherzolite xenoliths (Cala wava Volcanic District, central Spain). In: Celterti, M., Dewnes, H., GRÉGeIRE, M. \& O'ReILy, S. (eds) Petrological Evolution of the European Lithos pheric Mantle. Geological Society, London, Special Publications, 337, 107-124.

Benatti, E. \& Michael, P. J. 1989. Mantle peridotites from continental rif ts to ocean basins to subduction zones. Earth Planetary Science Letters, 91, 297-311.

BREY, G. P. \& KöHLER, T. 1994. Geothennobaromery in four-phase lherzolites; II, New thermobarometers, and practical assessment of existing thennobarometers. Joumal of Petrology, 31, 1353-1378.

Cebriá, J. M. \& Lórez-Ruiz, J. 1995. Alkali basalts and leucitites in an extensional in racontinental plate seting: the late Cenozoic Calatrava Volcanic Province (cenwal Spain). Lithos, 35, 27-46.

Chazet, G., Menzies, M. \& Harte, B. 1996. Silicate glasses in spinellherzolitef rom Yemen: origin and chemical composition. Chemical Geology, 134, 159-179.

Celterti, M., Beccaluva, L., Bønamman, C., Faccini, B., Ntafles, T. \& Siena, F. 204. Amphibole genesis via metasomatic reaction with clinopyroxene in mantle xenoliths from Victoria Land, Antarcica. Lithos, 75, 115-139.

Celterti, M., Beccaluva, L., Benamiman, C., Salvini, L. \& SIENA, F. 2000. Glasses in mantle xenoliths as 
geochemical indicators of metasomatic agents. Earth Planetary Science Letters, 183, 31-320.

Celterti, M., Benaøiman, C., Faccini, B., Grégerre, M., O'Reilly, S. Y. \& Powell, W. 2007a. Amphiboles from suprasubduction and intraplate lithosperic mantle. Lithos, 99, 68-74

Celterti, M., Benamiman, C., Faccini, B., Ntafles, T. \& SIENA, F. 2007b. Slab melt and in waplatemetasomaism in Kapfenstein mantle xenoliths (Styrian Basin, Auswia). Lithos, 94, 66-89.

Celterti, M., Bønamman, C., Hintøn, R. W., Siena, F. \& Urten, B. G. J. 1999. Carbonate metasomatism of the oceanic upper mantle: evidence from clinopyroxenes and glasses in ulwamafic xenoliths of Grande Comore, Indian Ocean. Journal of Petrology, 40, 133-165.

Demény, A., Vennemann, T. W. et AL. 2004. Trace element and $\mathrm{C}-\mathrm{O}-\mathrm{Sr}-\mathrm{Nd}$ isotope evidence for subduction-related carbonate-silicate melts in mantle xenoliths (Pannonian Basin, Hungary). Lithos, 75, 89-113.

DewnES, H. 201. Formation and modification of the shallow sub-continental lithospheric mantle: a review of geochemical evidence from ul ramafic xenolith suites and tectonically emplaced ultramafic massifs of western and central Europe. Journal of Petrology, 42, 233-250.

Dewnes, H., Reichew, M. K., Masen, P. R. D., Beard, A. D. \& ThIRlwall, M. F. 2003. Mantle domains in the lithosphere beneath the French Massif Cen wal: wace element and isotopic evidence from mantle clinopyroxenes. Chemical Geology, 200, 71-87.

DraPER, D. S. \& GreEN, T. H. 1997. P-T phase realions of silicic, alkaline, aluminous mantle-xenoliths glasses under anhydrous and $\mathrm{C}-\mathrm{O}-\mathrm{H}$ fluid saturated conditions. Joumal of Petrology, 38, 1187-1224.

Fernàndez, M., Marzán, I., Cerreia, A. \& Ramalhe, E. 1998. Heat flow, heat production, and lithospheric thennal regimen in the Iberian Peninsula. Tectonophysics, 291, 29-53.

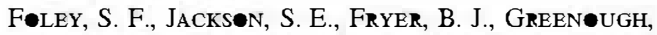
J. D. \& Jenner, G. A. 1996. Trace element partition coefficients for clinopyroxene and phlogopite in an alkaline lamprophyre from Newfounland by LA-ICPMS. Geochimica et Cosmochimica Act a, 60, 629-638.

Frey, F. A., Suen, C. J. \& Steckman, H. W. 1985. The Ronda high temperature peridotite: geochemical and petrogenesis. Geochimic et Cosmochimica Act 49, 2469-2491.

Galán, G., Oliveras, V. \& Patersen, B. A. 2008. Types of metasomatism in mantle xenoliths enclosed in Neogene-Qaternary alkaline mafic lavas from Catalonia (NE Spain). In: CelterTI, M. \& GRÉG@IRE, M. (eds) Metasomatism in ceanic and Continental Lithospheric Mantle. Geological Society, London, Special Publications, 293, 121-153.

Granet, M., Win.sen, M. \& Achauer, U. 1995. Inaging a mantle plume beneath the French Massif Cen wal. Earth Planetary Science Letters, 136, 281-296.

HarT, S. R. \& DunN, T. 1993. Experimental cpx/melt partitioning of 24 trace element. Contributions to Mineralogy and Petrology, 113, 1-8.

Hauri, e. H., Shimizu, N., Dieu, J. J. \& Hart, S. R. 1993. Evidence for hotspot-related carbonatite metasomatism in the oceanic upper mantle. Nature, 365, 221-227.

Ishimaru, S. \& ARAI, S. 2009. Highly silicic glasses in perido xenoliths from Avacha volcano, Kamchatka arc; implications for meling and metasomatism within sub-arc mantle. Lithos, 107, 93-106.

IsmaIl, M., Delpech, G., Cettin, J. Y., GréGerre, M., Meine, B. N. \& BILAL, A. 2008. Perological and geochemical constraints on the composition of the lithospheric mantle beneath the Syrian rift, northern part of the Arabian plate. In: CelterTI, M. \& GrÉGerRe, M. (eds) Metasomatism in ceanic and Continental Lithospheric Mantle. Geological Society, London, Special Publications, 293, 223-251.

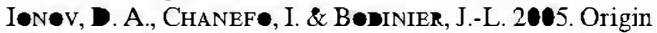
of Fe-rich lherzolites and wehrlites from Tok, SE Siberia by reactive melt percolation in refractory mantle peridotites. Contributions to Mineralogy and Petrology, 150, $335-353$.

la tøurrette, T., Hervig, R. L. \& Hølløway, J. R. 1995. Trace element partitioning between amphibole, phlogopite, and basanite melt. Earth Planetary Science Letters, 135, 13-30.

Le Bas, M. J., Le Maitre, R. W., Strecheisen, A. \& ZANETIN, B. A. 1986. Chemical classification of volcanic rocks based in the total alkali-silica diagram. Joumal of Petrology, 27, 745-750.

Lérez-Ruiz, J., Cebriá, J. M. \& Deblas, M. 2022. Cenozoic volcanism I: the Iberian peninsula. In: GibBens, W. \& Merene, T. (eds) The Geology of Spain. Geological Society, London, 417-438.

lépez-Ruiz, J., Cebriá, J. M., Deblas, M., Oyarzun, R., Heyes, M. \& Martín, C. 1993. The late Cenozoic alkaline volcanism of the cenwal Iberian Peninsula (Calawava Volcanic Province, Spain): in raplate volcanism realte to extensional tectonics. Journal of the Geological Society, London, 150, 915-922.

Lustrine, M. \& Wחisen, M. 2007. The circumMediterranean anorogenic Cenozoic igneous province. Earth Science Reviews, 81, 1-65.

MCDeneugh, W. F. \& Sun, S. 1995. The composition of the Earth. Chemical Geology, 120, 223-253.

Nimis, P. \& Ulmer, P. 1998. Clinopyroxene geobarometry of magmatic rocks Part 1: an expanded suctural geobarometer for anhydrous and hydrous, basic and ulwabasic systems. Contributions to Mineralogy and Petrology, 133, 122-135.

NiU, Y. 1997. Mantle melting and melt exraction processes beneath ocena ridges: evidence from abyssal peridotes. Journal of Petrology, 38, 1047-1074.

Nixøn, P. H. 1987. Mantle Xenoliths. Wiley, Chichester.

Nerman, M. D. 1998. Meling and metasomatism in the continental lithosphere: laser ablation ICPMS anlysis of minerals in spinel lherzolites from eastern Aus ralia. Contributions to Mineralogy and Petrology, 130, $24-255$.

Orejana, D., Villaseca, C. \& Patersen, B. A. 2006. Geochemistry of pyroxenitic and homblendic xenoliths in alkaline lamprophyres from the Spanish Central System. Lithos, 86, 167-196.

Orejana, D. \& Villaseca, C. 2008. Heterogeneous metasomatism in cumulate xenoliths from the Spanish Cen System: implications for percolative fractional crystallization of lamprophyric melts. 
In: Celterti, M. \& GRÉG@ire, M. (eds) Metasomatism in ceanic and Continental Lithospheric Mantle. Geological Society, London, Special Publications, 293, 11-12

Ossan, A. 1889. Beitrage zur kemtniss der eruptivegesteine des Capo de Gata (Almería). Zeitschrift der Deutschen Geologischen Gesellschafi, 51, 306-311.

Pearce, J. A., Barker, P. F., Emwards, S. J., Parkinsen, I. J. \& LEAT, P. T. 2000. Geochemisy and tectonic significance of peridotites from the South Sandwich arc-basin systems, south Atlanic. Contributions to Mineralogy and Petrology, 139, 36-53.

Pearsen, D. G., Canil, D. \& ShIrey, S. B. 2005. Mantle samples included in volcanic rocks: xenoliths and diamonds. In: CARLSeN, R. W. (ed.) The Mantle and Core. Treatise on Geochemistry, 2, 171-275.

Perinelli, C., Orlande, A., Cente, A. M., Armienti, P., Berrini, D., Faccini, B. \& Misiti, V. 2008 a. Metasomatism induce by alkaline magma in the upper mantle of northern Victoria Land (Antarcica): an experimental approach. In: ColTerTI, M. \& GRÉGerRe, M. (eds) Metasomatism in ceanic and Continental Lithospheric Mantle. Geological Society, London, Special Publications, 293, 279-312.

Perinelli, C., Safienza, G. T., Armienti, P. \& Merten, L. 2008b. Metasomatism of the upper mantle beneath the Hyblean Plateau (Sicily): evidence from pyroxenes and glass in peridotite xenoliths. In: CelTerTI, M. \& GrÉGerRE, M. (eds) Metasomatism in ceanic and Continental Lithospheric Mantle. Geological Society, London, Special Publications, 293, 197-221.

Piremalle, C., Gasperini, D., Macera, P. \& Faccenna, C. 2008. A late-Cretaceous contamination episode of the European-Mediterranean mantle. Earth and Planetary Science Letters, 268, 15-27.

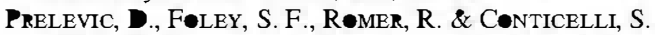
2008. Mediterranean teriary lamproites derived from multiple source components in postcollisional geodynamics. Geochimica et Cosmochima Acta, 72, 2125-2156.

Reyes, J., Villaseca, C., Barbere, L., Quejme, A. J. \& SAntes, J. F. 1997. Descripción de un método de separación de $\mathrm{Rb}, \mathrm{Sr}, \mathrm{Sm}$ y $\mathrm{Nd}$ en rocas silicatadas para estudios isotópicos. Congreso lbérico de Geoquímica, I, 46-55

San Miguel de la Cámara, M. 1936. Estudio de las rocas eruptivas de España. Memoria Academia de Ciencias de Madrid (Ciencias Naturales), 6.

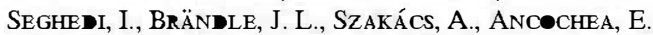
\& VASELLI, O. 2022. El manto litosférico en el sureste de España: Aportaciones de los xenolitos englobados en rocas alcalinas del Mioceno-Plioceno. Geogaceta, 32, 27-30

Shaw, C. S. J. \& Dingwell, D. B. 2018. Experimental peridovite-melt reaction at one atmosphere: a textural and chemical study. Contributions to Mineralogy and Petrology, 155, 199-214.

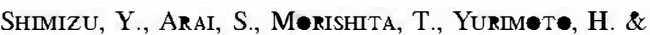
Gervilla, F. 2004. Petrochemical characteristics of felsic veins in mantle xenoliths from Tallante (SE Spain): an insight into activity of silicic melt within the mantle wedge. Transactions of the Royal Society of Edinburgh: Earth Sciences, 95, 265-276.

Takazawa, E., Frey, F. A., Shimizu, N. \& Obata, M. 2000. Whole rock compositional variations in an upper mantle peridotite (Homnan, Hokkaido, Japan): Are they consistent with a partial meling process? Geochimica et Cosmochimica Act a, 64, 695-716.

Teuren, S., Renac, C., O'Renly, S. Y., Cettin, J. Y. \& Griffin, W. L. 208. Characterization of the metasomatic agent in mantle xenoliths from Devès, Massif Central (France) using coupled in situ race-element and $\mathrm{O}, \mathrm{Sr}$ and $\mathbf{d}$ isotopic compositions. In: CeLTeRTI, M. \& GRÉG IRE, M. (eds) Metasomatism in Oceanic and Continental Lithospheric Mantle. Geological Society, London, Special Publications, 293, 177-196.

Uysal, I., Kaliweda, M., Karsli, O., Tarkian, M., Sadikzar, M. B. \& Ottley, C. J. 2007. Compostitional variations as a result of partial meling and melt-peridotite interaction in an upper mantle section from the Ortaca area, SW Turkey. Canadian Mineralogist, 45, 1471-1493.

Villaseca, C., Orejana, D., Patersen, B. A., BillSTrem, K. \& PÉrez-S•BA, C. 2007. Metaluminous pyroxene-bearing granulite xenoliths from the lower coninental crust in central Spain: their role in the genesis of Hercynian I-type granites. European Joumal of Mineralogy, 19, 463-477.

WitT-Eickschen, G. \& KRAMM, U. 1997. Mantle upwelling and metasomatism beneath central Europe: geochemical and isotopic constraints from mantle xenolithsf rom the Rhön (Gennany). Joumal of Petrology, 38, 479-493.

WeeD, B. J. \& BANNe, S. 1973. Garnet-orthopyroxene and Orthopyroxene-clinopyroxene relationships in simple and complex systems. Contributions to Mineralogy and Petrology, 42, 109-124.

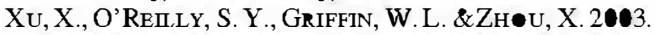
Enrichment of upper mantle peridovite: perological, trace element and isotopic evidence in xenoliths from SE China. Chemical Geology, 198, 163-188.

YAXLEY, G. M. \& KAMENETSKY, V. 1999. Insitu origin for glass in mantle xenoliths from southeastern Aus alia: insight from wace element compositions of glasses and metasomatic phases. Earth and Planetary Science Letters, 172, 97-109.

Yaxley, G. M., Kamenetsky, V., Green, D. H. \& FALL@N, T. J. 1997. Glasses in mantle xenoliths from western Victoria, Auswalia, and their relevance to mantle processes. Earth and Planetary Science Letters, 148, 433-446.

Zindler, A. \& HART, S. R. 1986. Chemical geodynamics. Annual Review of Earth and Planetary Sciences, 14, 493-571. 